\title{
Evolution of cis-regulatory modules for the head organizer gene goosecoid in chordates: comparisons between Branchiostoma and Xenopus
}

\author{
Yuuri Yasuoka ${ }^{1,2,3^{*}}$, Yukiko Tando ${ }^{4,5}$, Kaoru Kubokawa ${ }^{4,6}$ and Masanori Taira ${ }^{1,7}$
}

\begin{abstract}
Background: In cephalochordates (amphioxus), the notochord runs along the dorsal to the anterior tip of the body. In contrast, the vertebrate head is formed anterior to the notochord, as a result of head organizer formation in anterior mesoderm during early development. A key gene for the vertebrate head organizer, goosecoid (gsc), is broadly expressed in the dorsal mesoderm of amphioxus gastrula. Amphioxus gsc expression subsequently becomes restricted to the posterior notochord from the early neurula. This has prompted the hypothesis that a change in expression patterns of gsc led to development of the vertebrate head during chordate evolution. However, molecular mechanisms of head organizer evolution involving gsc have never been elucidated.
\end{abstract}

Results: To address this question, we compared cis-regulatory modules of vertebrate organizer genes between amphioxus, Branchiostoma japonicum, and frogs, Xenopus laevis and Xenopus tropicalis. Here we show conservation and diversification of gene regulatory mechanisms through cis-regulatory modules for gsc, $\lim 1 / 1 \mathrm{hx} 1$, and chordin in Branchiostoma and Xenopus. Reporter analysis using Xenopus embryos demonstrates that activation of gsc by Nodal/ FoxH1 signal through the $5^{\prime}$ upstream region, that of $\lim 1$ by Nodal/FoxH1 signal through the first intron, and that of chordin by Lim1 through the second intron, are conserved between amphioxus and Xenopus. However, activation of gsc by Lim1 and Otx through the 5' upstream region in Xenopus are not conserved in amphioxus. Furthermore, the 5' region of amphioxus gsc recapitulated the amphioxus-like posterior mesoderm expression of the reporter gene in transgenic Xenopus embryos.

Conclusions: On the basis of this study, we propose a model, in which the gsc gene acquired the cis-regulatory module bound with Lim1 and Otx at its 5' upstream region to be activated persistently in anterior mesoderm, in the vertebrate lineage. Because Gsc globally represses trunk (notochord) genes in the vertebrate head organizer, this cooption of gsc in vertebrates appears to have resulted in inhibition of trunk genes and acquisition of the head organizer and its derivative prechordal plate.

Keywords: Spemann-Mangold organizer, Gene regulatory network, Genomics, Vertebrate, Cephalochordate, Nodal/ FoxH1 signaling, Lim1, Otx, Chordin

\footnotetext{
* Correspondence: yuuri.yasuoka@riken.jp

${ }^{1}$ Department of Biological Sciences, Graduate School of Science, University of

Tokyo, 7-3-1 Hongo, Bunkyo-ku, Tokyo 113-0033, Japan

${ }^{2}$ Marine Genomics Unit, Okinawa Institute of Science and Technology

Graduate University, 1919-1 Tancha, Onna-son, Okinawa 904-0495, Japan

Full list of author information is available at the end of the article
}

(c) The Author(s). 2019 Open Access This article is distributed under the terms of the Creative Commons Attribution 4.0 International License (http://creativecommons.org/licenses/by/4.0/), which permits unrestricted use, distribution, and reproduction in any medium, provided you give appropriate credit to the original author(s) and the source, provide a link to the Creative Commons license, and indicate if changes were made. The Creative Commons Public Domain Dedication waiver (http://creativecommons.org/publicdomain/zero/1.0/) applies to the data made available in this article, unless otherwise stated. 


\section{Background}

The Phylum Chordata, named for the presence of the notochord on the dorsal side during embryogenesis, consists of three subphyla: Cephalochordata, Urochordata, and Vertebrata. Cephalochordates (amphioxus) are so named because the notochord extends to the anterior tip of the body ("cephalo-" denotes "head"). In the Vertebrata, the head is formed anterior to the notochord, suggesting evolutionary development of a "new head" in the space anterior to the notochord $[1,2]$. This head includes two lobes of the telencephalon, paired eyes, placodes, and cranial neural crest cells, which are lacking in amphioxus [3, 4]. Urochordates (tunicates) are quite diverse in morphology, despite their phylogenetic position as a vertebrate sister group. Therefore, amphioxus is a basal invertebrate chordate and the ancestral vertebrate was thought to be amphioxus-like [3, 4]. Thus, comparisons of developmental systems between amphioxus and vertebrates have provided important insights into the evolution of the vertebrate head. Although the definition of the vertebrate head remains problematic and involves many anatomical features, such as nerves, skeletal elements, and muscles, here we simply characterize the vertebrate head as the anteriorly enlarged central nervous system (forebrain) derived from anterior neuroectoderm, which is formed during gastrulation.

To date, based on comparative analyses of gene expression patterns and neuroanatomy, the rostral part of amphioxus central nervous system is postulated to be homologous to that of vertebrates, suggesting that the vertebrate brain was acquired on a foundation already present in the ancestral chordate, instead of through addition of a new part anterior to the notochord [5]. Even in hemichordates, a non-chordate deuterostome lineage, gene expression patterns along the anteroposterior (AP) axis in ectoderm are similar to those in vertebrate central nervous system, suggesting a deep ancestry of the molecular networks underlying AP axis formation in deuterostome nervous systems [6, 7]. In addition, abundant molecular evidence suggests that the lateral plate ectoderm of tunicates shares evolutionary origin with vertebrate neural crest and cranial placodes [8]. These recent molecular data argue strongly against the "new head" theory, which asserts that neural crest and neurogenic placodes are unique to vertebrates, and that most of the vertebrate forebrain are neomorphic structures $[1,2]$. Therefore, we need to investigate how the head region was converted from the amphioxus-type to the vertebrate-type. To address this question, we focused on head organizer genes in vertebrates, ot $x 2$, $\lim 1$ (also called $\operatorname{lh} x 1$ ), goosecoid (gsc), and chordin (chrd), by comparing their gene regulatory networks (GRNs) during head formation in frogs (Xenopus) and amphioxus.
In vertebrate embryogenesis, the head region is induced during the gastrula stage by the head organizer, which is the anterior part of the gastrula organizer (the Spemann-Mangold organizer in amphibians, the shield in teleosts, and the mid-gastrula organizer in mice). It should be noted that, in mouse embryos, anterior visceral endoderm is also involved in the anterior patterning despite the absence of axis inducing activity [9]. To understand head evolution in chordates, we focused on molecular mechanisms underlying head organizer formation, following gastrula organizer formation. In Xenopus and zebrafish, the gastrula organizer is formed in the late blastula stage by maternal canonical Wnt signaling from the dorsal region and Nodal signaling from the dorsovegetal region, which induce so-called organizer genes encoding transcription factors such as Otx2, Lim1, and Gsc, as well as Bmp antagonists such as Noggin, Chrd, and Cerberus involved in dorso-ventral (DV) patterning [10-13]. During gastrulation, the organizer is gradually divided into head and trunk organizers, which promote antero-posterior (AP) patterning of neuroectoderm $[14,15]$. The head organizer induces forebrain and midbrain formation and also determines the anterior midline [16]. Head and trunk organizer regions develop into the prechordal plate and the notochord, respectively. The prechordal plate is a vertebrate-specific tissue that escapes from convergent extension movements occurring in notochordal cells.

The homeobox gene gsc is known as a head organizerspecific and later, a prechordal plate-specific gene, and is necessary for repressing trunk organizer genes and ventral genes such as brachyury, wht8, and ventx genes [17-21]. The trunk organizer gene, brachyury, is a crucial gene for notochord formation [22]. Knockdown analysis of gsc in Xenopus embryos results in a short head and a cyclops phenotype, caused by reduction of the prechordal plate [20, 21]. A recent genome-wide study by our group using Xenopus tropicalis embryos also showed that Gsc binds to thousands of genomic regions and represses many trunk genes in concert with Otx 2 and TLE/Groucho corepressor in the head organizer of early gastrula embryos [21]. Thus, Gsc is thought to be a key regulator of the head organizer and of prechordal plate development.

In amphioxus, recent extensive studies of expression patterns of many developmental regulatory genes and roles of cellular signaling during early embryogenesis have shown that fundamental mechanisms of DV and AP axis formation and expression patterns of organizer and nonorganizer genes are highly conserved between amphioxus and vertebrates [23-26]. For example, chrd is expressed in dorsal mesoderm and ectoderm [24]. The LIM homeobox gene $\lim 1 / 5$ (an ortholog of vertebrate paralogs $\lim 1$ and lim5) is expressed in dorsal mesoderm (lim1 type) and ectoderm (lim5 type) [27]. The homeobox gene otx is 
expressed in endomesoderm and anterior ectoderm in amphioxus gastrulae [25]. Expression patterns of these genes in amphioxus are quite similar to those of their orthologs in Xenopus (Fig. 1a) [28-35]. However, gsc displays a different pattern in amphioxus; its expression starts in the gastrula organizer during the early gastrula stage, and remains active throughout axial mesoderm (presumptive notochord domain) until the early neurula stage [24]. It is finally restricted to the posterior end of the notochord from early to mid-neurula stage [36] (Fig. 1a). In contrast, in vertebrates, $g s c$ is expressed in the head organizer region at the late gastrula, and later in the prechordal plate, but not in the notochord [35, 37]. Based on these observations, Neidert et al. (2000) hypothesized that the shift of gsc expression to the anterior mesoderm during late gastrula stages is an important evolutionary event in the innovation of the head organizer, which later differentiates into the prechordal plate. However, the underlying regulatory mechanisms that enabled the change of gsc expression during vertebrate evolution remain largely unknown.
In Xenopus embryos, gsc is first expressed in the gastrula organizer under the influence of Wnt and Nodal signaling through the cis-regulatory module (CRM) located near the gsc promoter region, named gsc-U1 [21, 35], which includes the proximal and distal elements (DE and PE) [38-41]. The Wnt-induced homeodomain protein, Siamois and Twin, mediates activation of gsc [42]. Nodal signaling is transduced by phosphorylation of $\operatorname{Smad} 2 / 3$, and phosphoSmad2/3 directly upregulates $g s c$ in concert with partner transcription factors FoxH1 and Wbscr11 [38, 43]. Its expression is subsequently maintained in the head organizer by Lim1 and Otx2 through the gsc-U1 [21, 35, 39]. In the posterior and ventral regions, gsc expression is repressed by the Bmp-activated, ventrally expressed, homeodomain transcription factor, Vent2 [44] and Vent1/PV.1 [39], through the same CRM, gsc-U1. Recently, reporter analyses using amphioxus genomic regions in mammalian cell lines and medaka fish have shown that activation of vent genes by Bmp and repression of chrd and gsc by Vent through their upstream regulatory regions is conserved
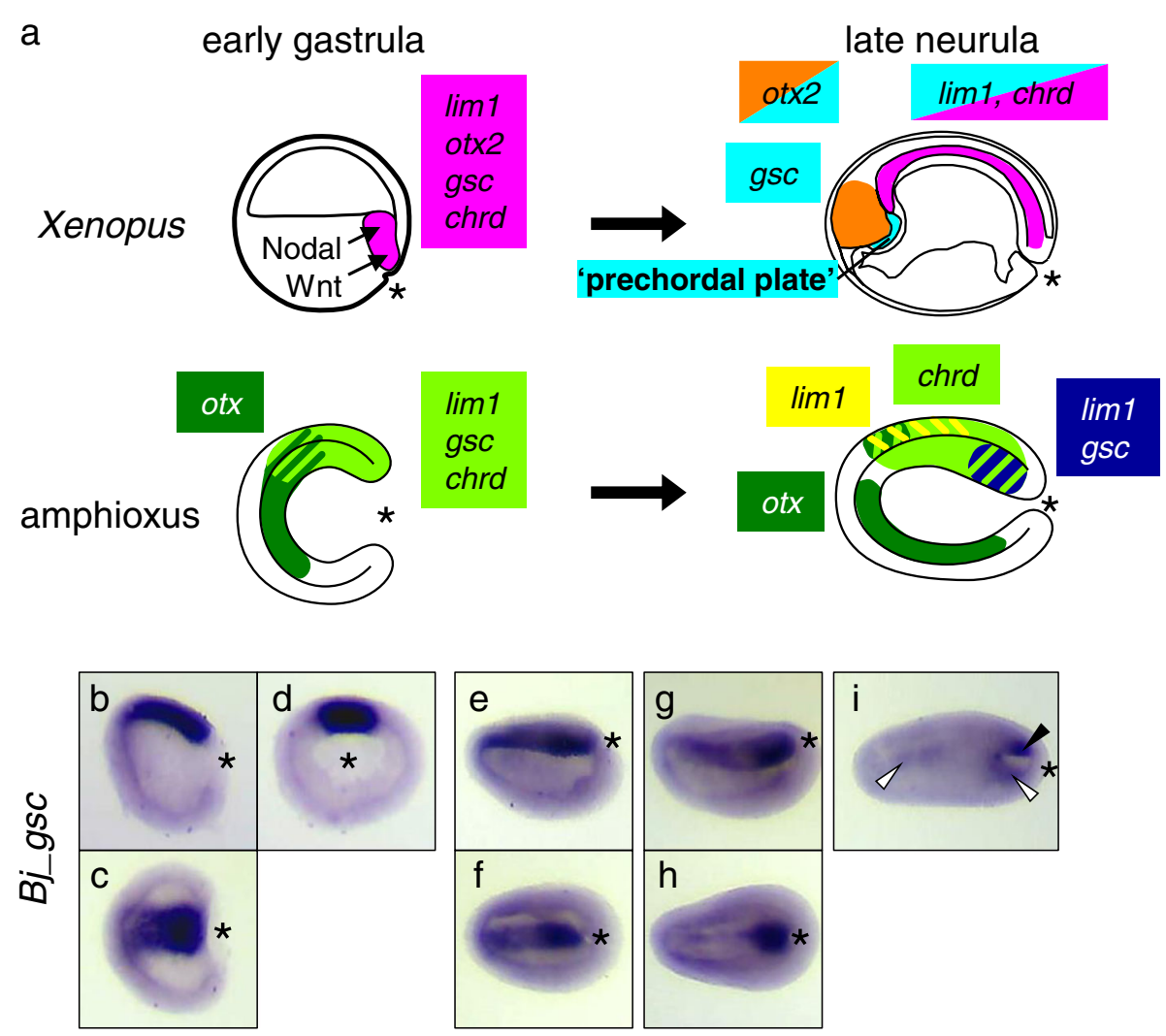

Fig. 1 Schematic representations of organizer gene expression patterns. a Expression patterns of lim 1, otx2 (otx in amphioxus), goosecoid (gsc), and chordin (chrd) of Xenopus (top) and amphioxus (bottom) at the early gastrula stage (left) and the late neurula stage (right) are shown with colors as indicated. b-i Whole-mount in situ hybridization of gsc in B. japonicum embryos. In mid-gastrula (stage G5-6), Bj_gsc is expressed in the dorsal mesoderm (b-d). In late gastrula (stage G7-N0), Bj_gsc is still expressed throughout the dorsal mesoderm (e, f). In early neurula (stage N1), Bj_gsc expression is still strong in the posterior mesoderm but very weak in the anterior mesoderm ( $\mathbf{g}, \mathbf{h}$ ). In mid-neurula (stage N2), Bj_gsc is expressed in the posterior mesoderm (arrowhead) and weakly in the dorsal endoderm (open arrowheads) (i). Embryos are shown in lateral view with dorsal to the top and anterior to the left $(B, \mathbf{e}, \mathbf{g}, \mathbf{i})$, dorsal view with anterior to the left $(\mathbf{c}, \mathbf{f}, \mathbf{h})$, or blastoporal view with dorsal to the top (d). ${ }^{*}$, blastopore 
between amphioxus and vertebrates $[45,46]$. However, although these analyses have shown conserved gene regulatory networks (GRNs) for patterning along the DV axis, it remained to be determined whether GRNs in the head and trunk organizers along the AP axis are conserved. Thus, we carried out comparative analyses of CRMs for gsc together with those for lim 1 and chrd, to compare the organizers in amphioxus and vertebrates using Xenopus embryos. The Xenopus embryo is a most representative vertebrate embryo for comparison of the gastrulation process and gene expression patterns in chordates [24, 47], as both Xenopus and amphioxus exert the holoblastic cleavage without extraembryonic tissues. Also, the large knowledge of the organizer has been accumulated by Xenopus studies, as described above. Here we discuss how the chordate head evolved.

\section{Results}

Bj_gsc expression is gradually restricted to posterior mesoderm during neurulation

Before analyzing CRMs, we reexamined expression patterns of gsc in amphioxus using B. japonicum embryos (Fig. 1b-i). As shown in previous studies using B. floridae, Bj_gsc is expressed throughout the dorsal mesoderm in mid-gastrula (stages G5-G6, Fig. 1b-d) and late gastrula (stage G7-N0, Fig. 1e, f). In early neurula (stage $\mathrm{N} 1$ ), Bj_gsc expression starts to be restricted to the posterior mesoderm, but remains weak in the anterior mesoderm (Fig. 1g, h). In mid-neurula (stage N2), mesodermal expression of $B j \_g s c$ is completely restricted to the posterior notochord (Fig. 1i), while Bj_gsc expression is detectable in anterior and posterior dorsal endoderm.

To avoid confusion, we should note that previous studies of gsc expression in B. floridae labeled embryonic stages incorrectly. First, Neidert et al. [36] described $B f_{-}$ gsc expression localized in posterior mesoderm at midgastrula stage but the embryo is early neurula (stage N1). Second, Neidert et al. [36] also mislabeled midneurula (stage N2) with seven pairs of somite as late gastrula. Third, Yu et al. [24] described Bf_gsc expression in the entire dorsal mesoderm at neurula stage, but the embryo appears to be at the late gastrula to pre-hatching neurula stage (stage G7-N0). Thus, our results are consistent with previous studies and clearly describe dynamic changes of gsc expression during neurulation of amphioxus embryos.

\section{Genomic sequences of $B j$ gsc, Bj_lim 1 and $B j$ chrd}

To compare CRMs of organizer genes between amphioxus and vertebrates, we cloned amphioxus counterparts of vertebrate CRMs for $g s c$, lim1, and $c h r d$, from Japanese amphioxus, Branchiostoma japonicum (Bj_gsc, Bj_ lim1, and Bj_chrd, respectively). Because vertebrate gsc is regulated through the conserved CRM, gsc-U1, which is located in the $0.5 \mathrm{~kb} 5^{\prime}$ region, we isolated the roughly
$4.5 \mathrm{~kb} 5^{\prime}$ region, including the promoter and $5^{\prime}$ untranslated region (UTR) sequence of $B j \_g s c$. We also isolated the first intron of $B j \lim 1$, because $\lim 1$ is activated by Nodal signaling in the organizer through the first intron in Xenopus and zebrafish $[48,49]$. For chrd, the previous study identified a CRM near the promoter (around 1.5 kb 5' flanking region) in Florida amphioxus, Branchiostoma floridae [45, 46]. However, CRMs for activation of chrd by Lim 1 and Otx 2 were not located in the promoter in Xenopus [21]. Therefore, we sought to identify conserved chrd CRMs.

We found enhancer activity of the second intron of Xenopus chrd (Xt_chrd, Xl_chrd.L, and Xl_chrd.S, Fig. 2a;. L and. $S$ indicate homeologs in subgenomes of the allotetraploid frog X. laevis [51]), which contains a CRM bound with Lim1 and Otx2, named chrd-D1 [21]. Luciferase reporter assays in Xenopus embryos showed that Lim1, with its cofactors Ldb1 and Ssbp3a, Otx2, Siamois and Nodal signaling, activate the reporter gene through the second intron in Xenopus (Fig. 2b-d). The non-responsiveness of a deletion construct which lacks a conserved FoxH1 binding motif to Nodal signaling suggests that Nodal/FoxH1 signaling directly regulates chrd (Fig. 2e). In fact, FoxH1 binds to chrd-D1 in Xenopus gastrulae [52]. In addition, a Lim1 binding motif mutated construct abolished the enhancer activity activated by Lim1/Ldb1/Ssbp3, indicating that Lim1 directly activates chrd through chrd-D1 (Fig. 2f). Furthermore, we also performed transgenic reporter assays using Xenopus embryos, resulting in activation of reporter gene expression in the head organizer of gastrulae through $X t$ chrd-D1, similar to the endogenous chrd (Fig. 2g). Based on these results, we cloned the second intron of $B j_{\text {cchrd }}$ as a counterpart of vertebrate CRM for evolutionary comparisons.

Cloned genomic sequences of $B j_{-} g s c$, $B j_{-} \lim 1$, and $B j_{-}$ chrd were then compared by Vista plot with the genome database of Branchiostoma floridae [53] (Bf_gsc, Bf_lim1 and Bf_chrd, respectively), Branchiostoma belcheri [54] (Bb_gsc, Bb_lim1 and Bb_chrd, respectively), and Branchiostoma lanceolatum [55] (Bl_gsc, Bl_lim1 and $B l_{-}$ chrd, respectively) to depict evolutionarily conserved regions as candidates of CRMs [56,57]. There are a number of conserved regions in each pair of the sequences, in which we found conserved binding motifs for Lim1 (T/CTAATT/GA/G), Otx2 (bicoid site, TAATCC/T), FoxH1 (TGTNNATT), and Smad (GTCTG) (Fig. 3). In addition, we surveyed epigenetic data of B. lanceolatum embryos [55] to find more plausible CRM candidates (Fig. 3). The data show that conserved non-coding regions among Branchiostoma species overlap closely with open chromatin regions, which correspond to ATAC-seq peaks, in B. lanceolatum embryos. We next tested CRM activities of these regions using Xenopus embryos, as described below. 

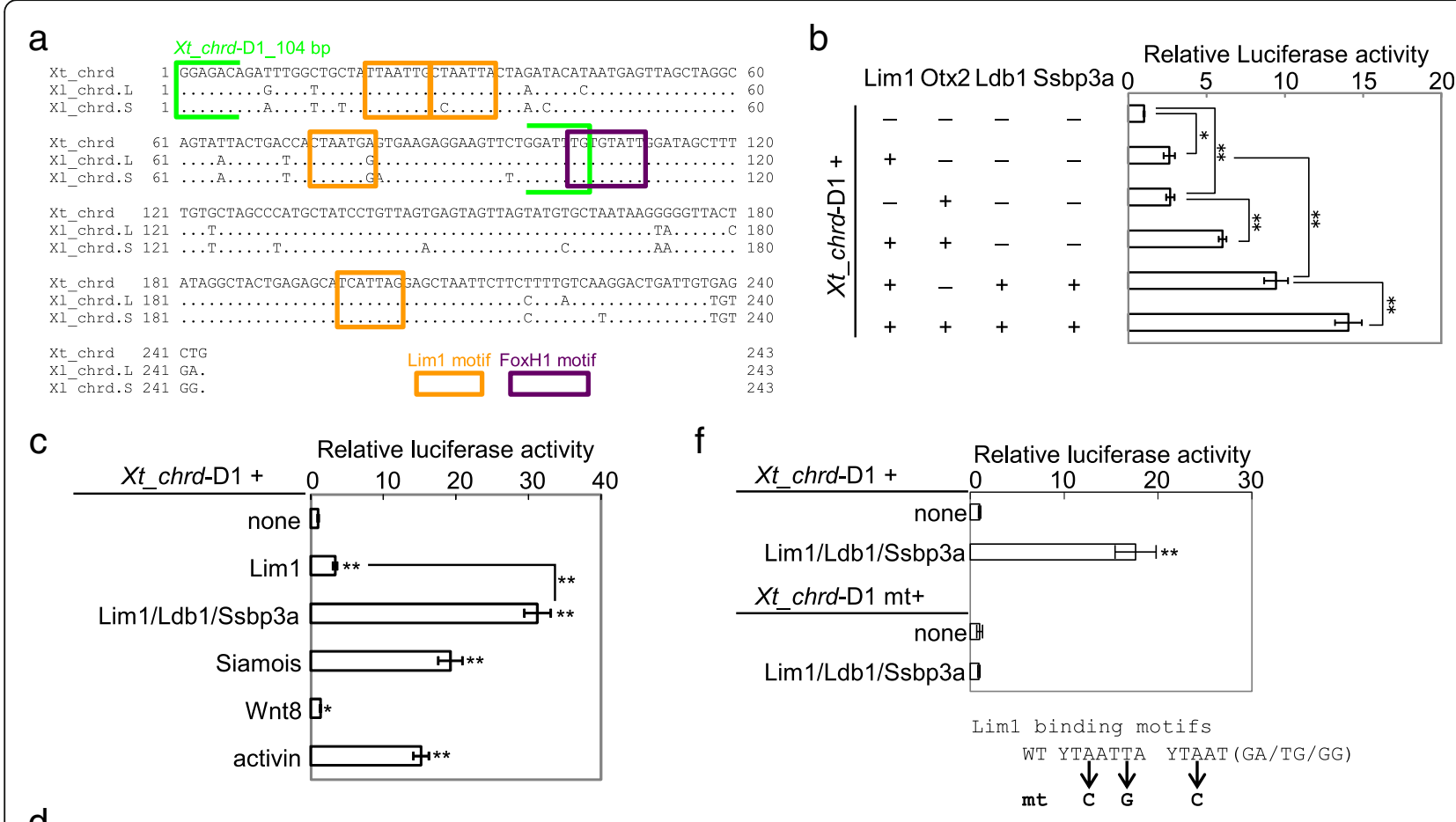

d

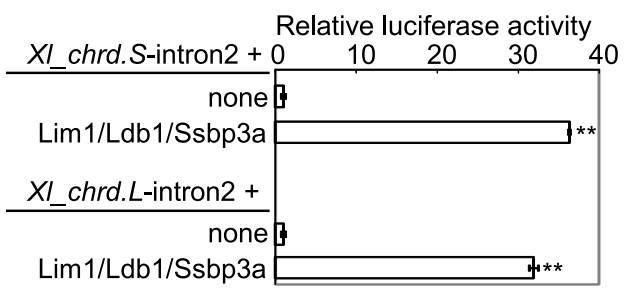

e

Relative luciferase activity
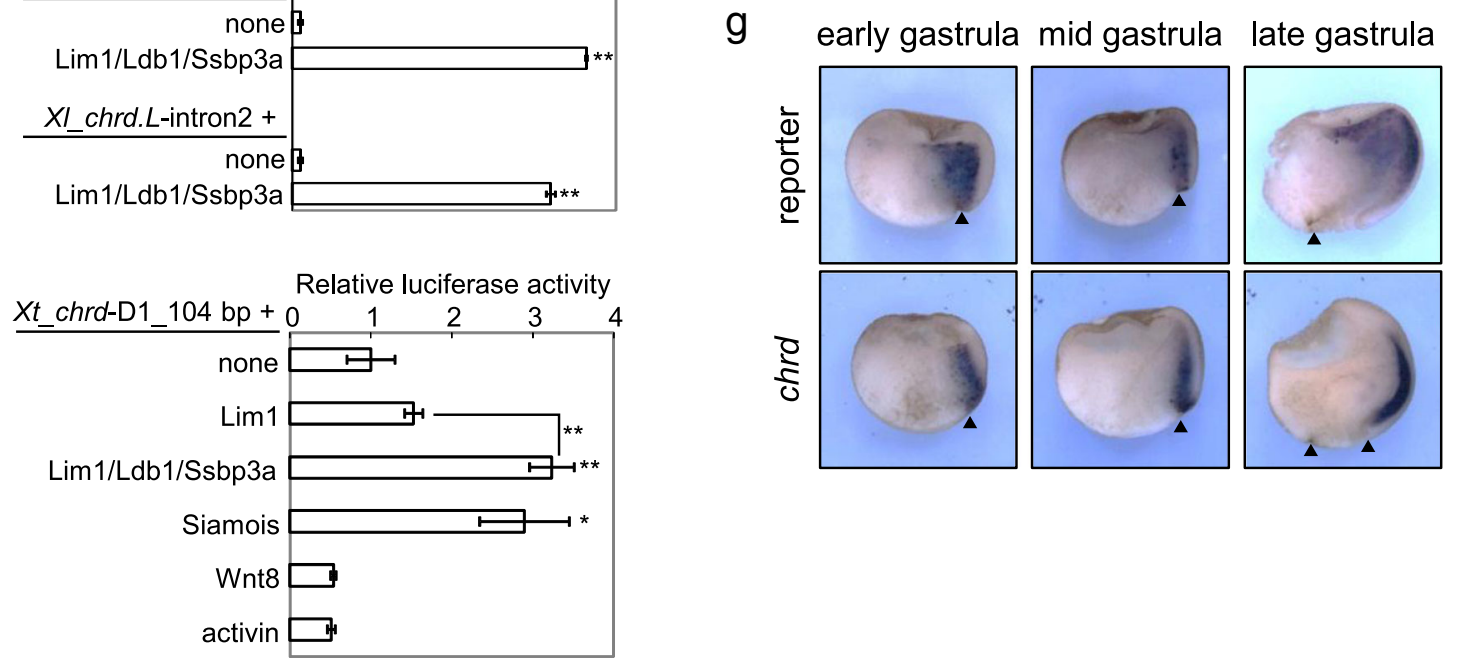

Fig. 2 CRM activities of the second intron of Xenopus chrd. a Sequence alignment of $X$. tropicalis and X. laevis chrd-D1 core regions (243 bp). Orange boxes, conserved Lim1 motifs; purple box, conserved FoxH1 motif (a major partner of Smad2/3 in Nodal signaling). Siamois may bind to the Lim1 site [50]. b-f Luciferase reporter assays. Xt_chrd-D1 (b, c), intron 2 sequences of XI_chrd.L and .S (d), Xt_chrd-D1_104 bp (between light green brackets in a) (e), and X__chrd-D1_mt (all four conserved Lim1 motifs are mutated) were analyzed. Xt_chrd-D1 showed synergistic activation by Lim1, Ldb1, Ssbp3, and Otx2 (b). Strong activation through Xt_chrd-D1 was observed in Lim1/Ldb1/Ssbp3, Siamois and activin, but not in Wnt8 (c). XI_chrd.L and.S intron2 showed conserved enhancer activity, which was activated by Lim1/Ldb1/Ssbp3 (d). No responsiveness of Xt_chrd -D1_104 bp to activin (e) suggests that Nodal signaling activates chrd-D1 through the conserved FoxH1 site. Reporter activation by Lim1/ Ldb1/Ssbp3 was abolished by mutating four Lim1 motifs in Xt_chrd-D1 (f), indicating that Lim1 directly activates chrd through the intron2 enhancer. Bars represent mean \pm s.e.m. ${ }^{*}, P<0.05 ;{ }^{* *}, P<0.01$ (t-test, two tailed). Dosages of injected mRNAs are as follows: lim1, 100 pg/embryo; Idb1, 100 pg/embryo; ssbp3, 100 pg/embryo; otx2, 40 pg/embryo; simois, 100 pg/embryo; wnt8a, 25 pg/embryo; and activin A, 20 pg/embryo. g Transgenic reporter analysis of Xt_chrd-D1. Panels represent whole mount in situ hybridization of the reporter gene mVenus or endogenous chrd for transgenic embryos with dorso-ventral hemisections. Expression patterns were examined at the early (st. 10), middle (st. 11) and late (st. 12.5) gastrula stages as indicated. In total, 8 of 30 transgenic embryos showed reporter expression in the organizer. Embryos are shown with the animal pole at the top and dorsal to the right. Arrow heads, blastopore 


\section{a}

Bl lim1 intron1

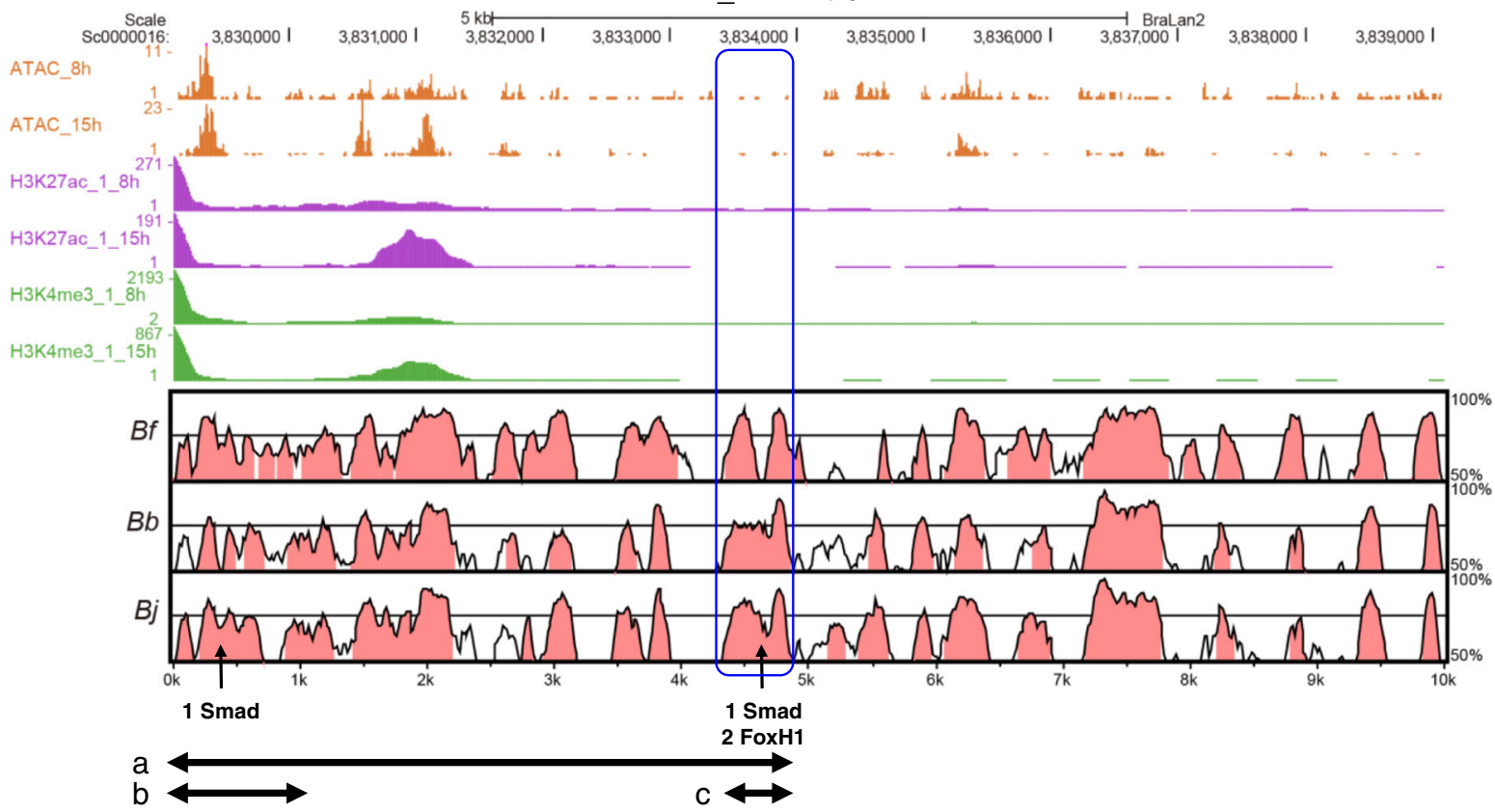

b

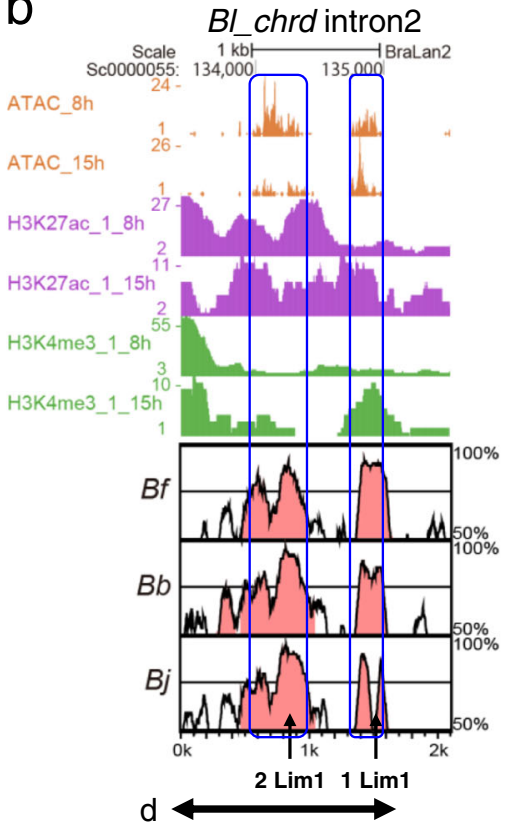

C

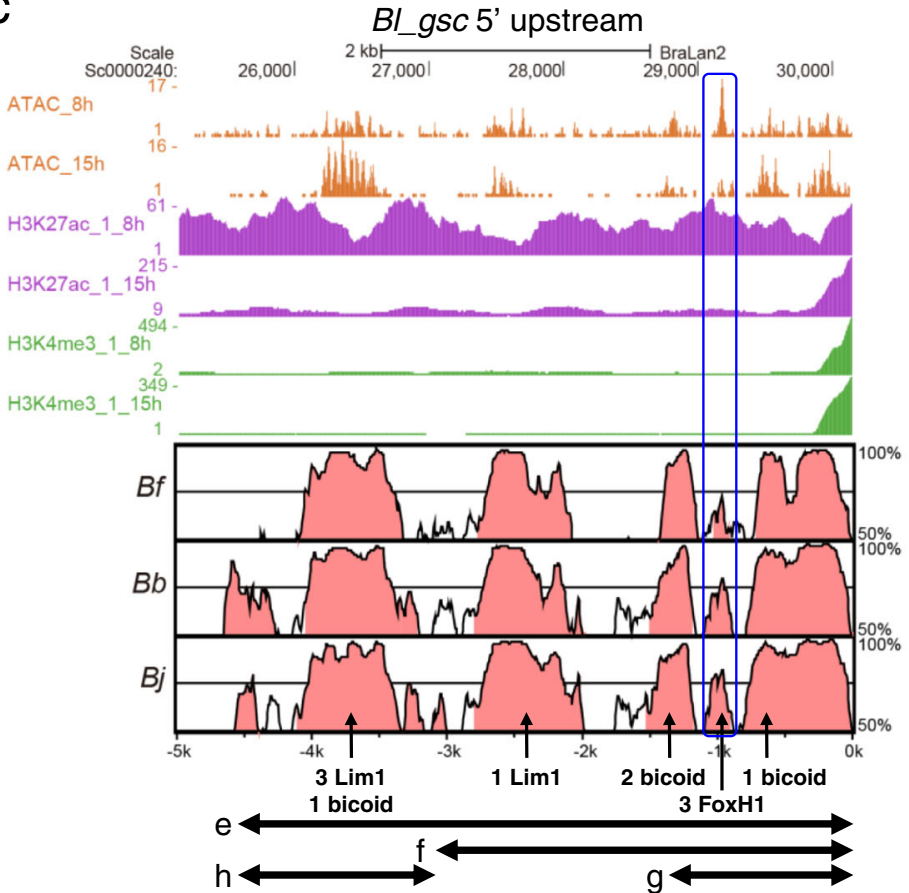

Fig. 3 Epigenetic data from B.lanceolatum embryos and sequence comparisons between B.lanceolatum, B. floridae, B. belcheri, and B. japonicum. a ATAC-seq, H3K27ac ChIP-seq, and H3K4me3 ChIP-seq data from early gastrula (8 hpf) and early neurula (15hpf) in Bl_lim 1 intron 1 are represented with Vista plot of BI_lim1 intron 1 vs Bf_lim1 intron 1, Bb_lim1 intron1 and Bj_lim1 intron 1. Regions with 50-100\% identity were shown and conserved non-coding sequences (CNSs) were colored in red. The number of Smad motifs and FoxH1 motifs in CNSs is shown as indicated by arrows. $\mathbf{b}$ Epigenetic data in BI_chrd intron 2 are represented with Vista plot of BI_chrd intron 2 vs Bf_chrd intron 2 Bb_chrd intron 2 and Bj_chrd intron 2. The number of Lim1 sites in CNSS is indicated by arrows. c Epigenetic data in BI_chrd intron 2 are represented with Vista plot of the -5 $\mathrm{kb}$ region of Bl_gsc vs those of Bf_gsc, Bb_gsc and Bj_gsc. The number of Lim1, bicoid, and FoxH1 sites in CNSs is shown as indicated by arrows. $a-h$, Regions used for reporter assays. Blue boxes indicate putative CRMs analyzed in reporter assays (Figs. 4 and 5). Additional file 1: Figure S1 shows sequence alignment of them (see Additional file 1) 


\section{Conservation of CRM activities of introns of Bj_lim 1 and Bj_chrd}

To examine the conservation of $\lim 1$ regulation by Nodal signaling, we performed luciferase reporter assays in Xenopus embryos using reporter constructs, in which the previously reported activin response element (ARE) in Xenopus lim 1 intron 1 (cloned from $X$. tropicalis, $X t$ lim1-D1) or Bj_lim1 intron 1 sequences were connected upstream of the minimum promoter (Fig. 4a). The $5 \mathrm{~kb}$ $5^{\prime}$ sequence of Bj_lim 1 intron 1 , but not the $1 \mathrm{~kb}$ sequence responds to Activin, which activates Nodal signaling (compare constructs $\mathrm{a}$ and $\mathrm{b}$ in Fig. 4a). This enhancer activity was detected in the $0.5 \mathrm{~kb}$ fragment at the 3' end of the 5' half of Bj_lim 1 intron 1 (construct c in Fig. 4a), which contains FoxH1 and Smad binding motifs. Mutation of FoxH1 binding motifs but not that of a Smad binding motif in the $0.5 \mathrm{~kb}$ fragment resulted in the absence of responsiveness to Nodal signaling, suggesting that Bj_lim1 is induced by Nodal signaling in the gastrula organizer through the FoxH1 binding sites at the middle of its intron 1 (Fig. 4b). Thus, regulation of lim 1 by Nodal signaling in early gastrula embryos appears to be conserved in chordates.

However, those FoxH1 binding motifs are not conserved among Branchiostoma, although surrounding sequences are conserved (Additional file 1: Figure S1A). In addition, ATAC-seq peaks and enhancer histone marks in early embryos were not detected in the genomic region of B. lanceolatum corresponding to Bj_lim 1 intron 1 ARE (Fig. 3a). Although there are ATAC-seq peaks around $2 \mathrm{~kb}$ from $5^{\prime}$ end of $B l \_l i m 1$ intron1, this region encodes a non-coding RNA gene (BL38158 gene) and enriched with promoter histone marks (H3K4me3 and H3K27ac). Therefore, some other regions may respond to Nodal signaling in B. lanceolatum, which should be tested in future.

To examine conservation of the organizer gene regulatory axis for chrd, we next performed reporter assays using reporter constructs of Bj_chrd intron 2 sequences. Bj_chrd intron 2 is activated by a combination of Lim1, Ldb1 and Ssbp3a, and mutations in Lim1 binding motifs reduced the responsiveness (Fig. $4 \mathrm{c}, \mathrm{d}$ ), similar to $X t$ chrd-D1 (Fig. 2b). Conservation of those Lim1 binding motifs among Branchiostoma species and epigenetic data of B. lanceolatum embryos further support the enhancer activity of chrd intron 2 during amphioxus development (Fig. 3b; Additional file 1: Figure S1B,C). This result suggests that regulation of chrd by Lim1 in early gastrula embryos is conserved in chordates. Although significant activation by Otx2 was observed in Xt_chrd-D1 (Fig. 2b), Otx2 could not significantly activate the Bj_chrd intron 2 reporter with Lim1/Ldb1/ Ssbp3a (Fig. 4c), implying a weaker effect of Otx on chrd expression in amphioxus.

\section{The $5^{\prime}$ region of $B j \_g s c$ responds to Wnt and nodal, but not Lim1 and Otx}

To address how amphioxus gsc is regulated, we carried out luciferase and transgenic reporter analyses for $\mathrm{Bj}_{-}$ gsc regulatory regions (Fig. 5). The data showed that the $-4.5 \mathrm{~kb},-3 \mathrm{~kb}$, and $-1.5 \mathrm{~kb} B j \_g s c$ regions (constructs e, f, and g, respectively) are activated strongly in the marginal zone at higher levels in the dorsal side than ventral side, although the $-1.5 \mathrm{~kb}$ region is not sufficient for a full response. On the other hand, the $4.5 /-3 \mathrm{~kb}$ region (construct $\mathrm{h}$ ) is weakly activated only in the dorsal marginal zone but not in the ventral side. This suggests that the $-3 \mathrm{~kb}$ region contains CRMs responding to some factors in the dorsal mesoderm. In the $-3 \mathrm{~kb}$ region, there are conserved noncoding sequences overlapped with ATAC-seq peaks in B. lanceolatum embryos, possibly corresponding to active enhancers (Fig. 3c). We next examined what factors activate this $-3 \mathrm{~kb}$ region in the dorsal mesoderm.

Lim1, Ldb1, and Ssbp3 strongly activated gsc-U1 of Xenopus (upper panel of Fig. 5b), but they and Otx2 could elicit no or only slight activation of the $-4.5 \mathrm{~kb}$ Bj_gsc reporter (middle panel of Fig. 5b), in spite of the presence of several conserved motifs for Lim1 and Otx2 (Fig. 3c). By contrast, activin strongly activates the Bj_gsc promoter (lower panel of Fig. 5b), as has been shown in $X l$ gsc $[38,40]$. Wnt8 weakly activates the Bj_gsc promoter and also weakly synergizes with activin. We think that Wnt signaling indirectly activates the promoter in Xenopus embryos (see Discussion). Reporter constructs with mutated FoxH1 binding motifs exhibited greatly reduced responsiveness to activin, suggesting that Nodal signaling directly activates $B j$ gss via FoxH1 binding on conserved motifs in the $5^{\prime}$ region (Fig. $5 c$; Additional file 1: Figure S1D). These results indicate that the Bj_gsc promoter is activated by organizer-inducing signals Wnt and Nodal, but not by the organizer transcription factors Lim1 and Otx2, implying that the difference in expression patterns between amphioxus and vertebrate gsc genes (Fig. 1 ) is attributable to the difference in the response to Lim1 and Otx2. However, it should be noted that these data do not exclude the possible presence of Lim1/Otxdependent CRMs that may regulate gsc expression in other genomic regions, and that the difference in gsc expression may not solely depend on the difference in CRMs analyzed here.

Transgenic reporter analyses using Xenopus embryos further clarified the difference in dorsal expression in gastrula and neurula (Fig. 6; Additional file 1: Figure S2). In the early gastrula stage, $\mathrm{a}-3 \mathrm{~kb}$ region of $X t$ gsc, which includes CRMs-U1 to U3 [21], and a $-4.5 \mathrm{~kb}$ region of Bj_gsc both showed reporter expression in the organizer region, as well as the endogenous $g s c$, suggesting that conserved responses to Nodal signaling result in 
a
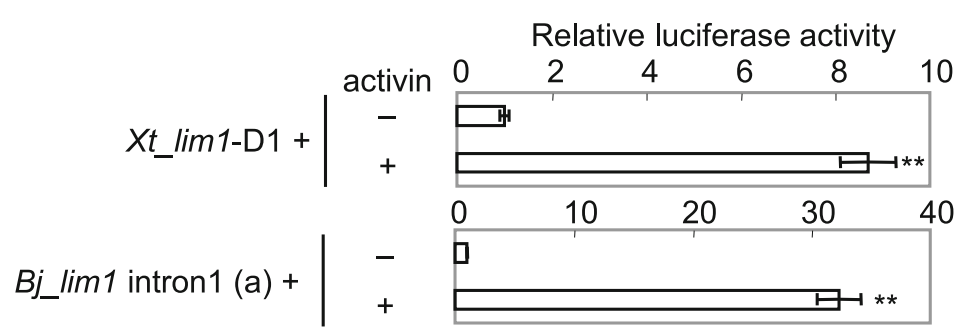

Bj_lim1 intron1 (b) $+\mid$\begin{tabular}{llllll|}
- & 0 & 10 & 20 & 30 \\
+ & $j$ & & & & \\
\hline & 0 & 10 & 20 & 30 & 40
\end{tabular}

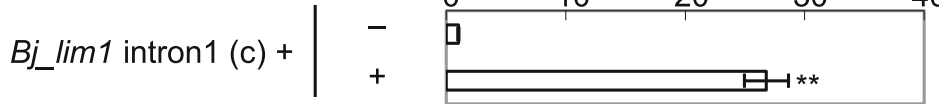

b

Relative luciferase activity

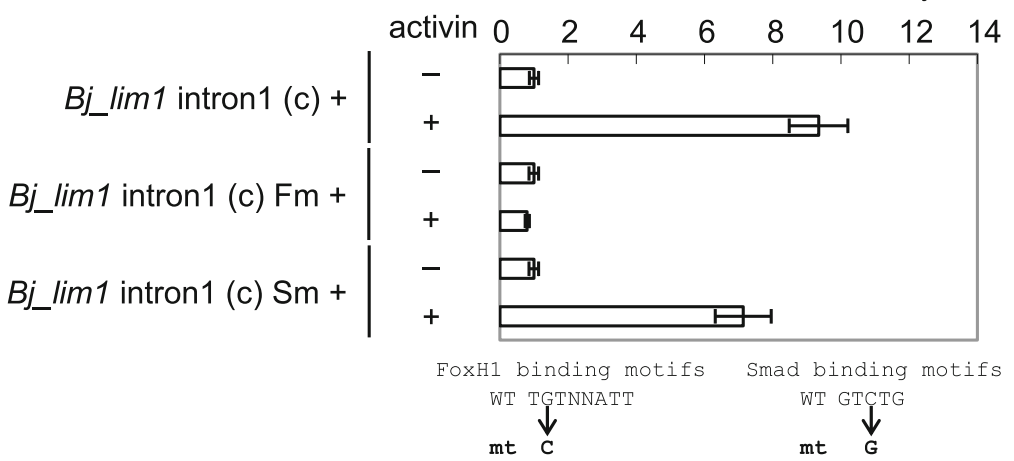

C

Lim1/Ldb1 Otx2 Relative luciferase activity

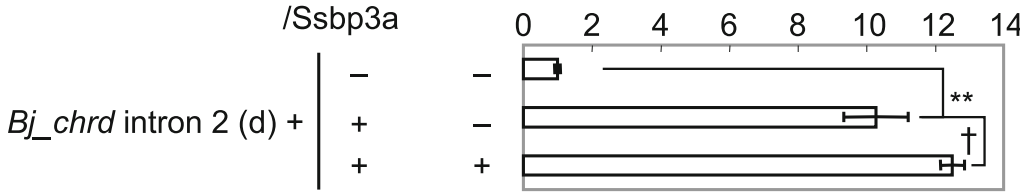

d

Lim1/Ldb1 Relative luciferase activity

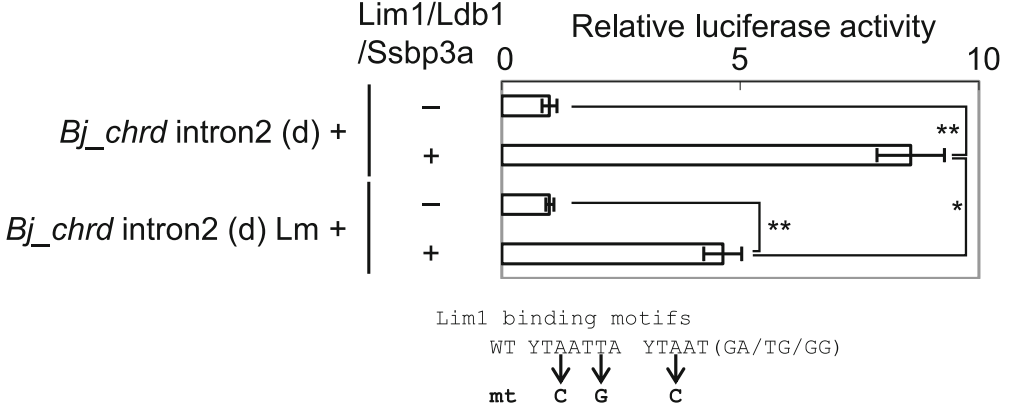

Fig. 4 Reporter analyses of the $\lim 1$ intron 1 and chrd intron 2 in the Xenopus embryo. a, b Luciferase reporter assays of $\lim 1$ intron 1. Responsiveness of reporter constructs to Nodal signaling was tested with or without activin A mRNA (40 pg/embryo). A reporter construct mutated in two FoxH1 motifs (Fm), but not that in a Smad motif ( $\mathrm{Sm}$ ) exhibited no response to Nodal signaling (b), suggesting that Nodal signaling directly regulates Bj_lim1 through FoxH1 binding to the intron1 enhancer. c, d Luciferase reporter assays of Bj_chrd intron 2.

Responsiveness of Bj_chrd intron 2 (region g) to Lim1 and Otx2 was tested with or without lim1, Idb1, ssbp3, and otx2 mRNAs (100, 100, 100 and 40 pg/embryo, respectively). Lim1/Ldb1/Ssbp3 significantly activated the reporter gene through Bj_chrd intron 2 (C), but the activation level was significantly reduced by mutating three Lim 1 motifs (D). See Fig. 2 for details of the Lim 1 motif mutation. Bars represent mean \pm s.e.m. ${ }^{*}, P<0.05$, **, $P<0.01 ;+, P<0.1$ ( $t$-test, two tailed) 
a Relative luciferase activity

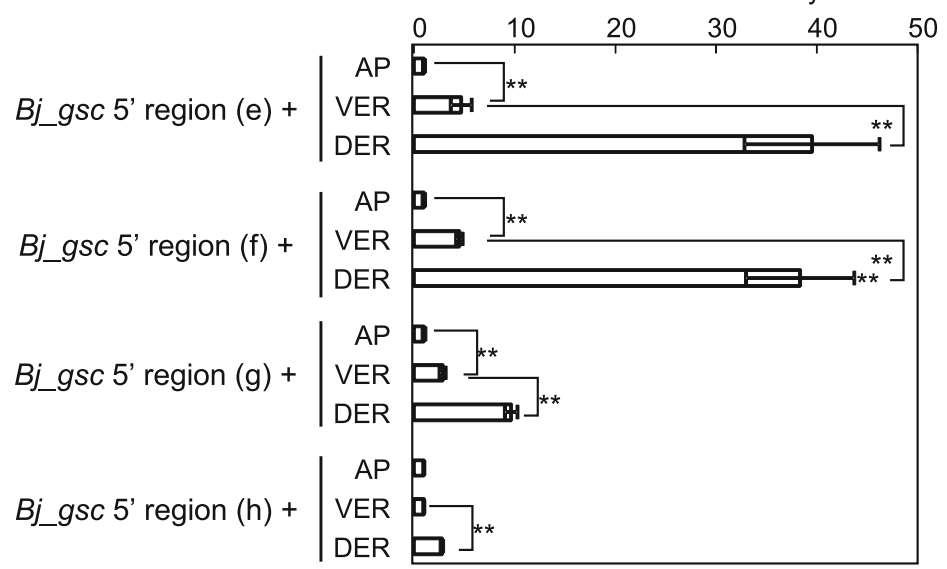

b

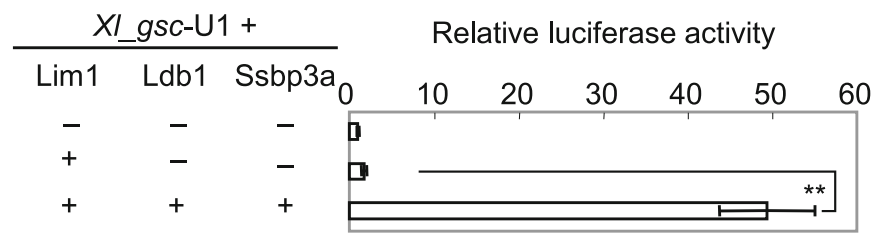

Bj_gsc 5' region (e)+

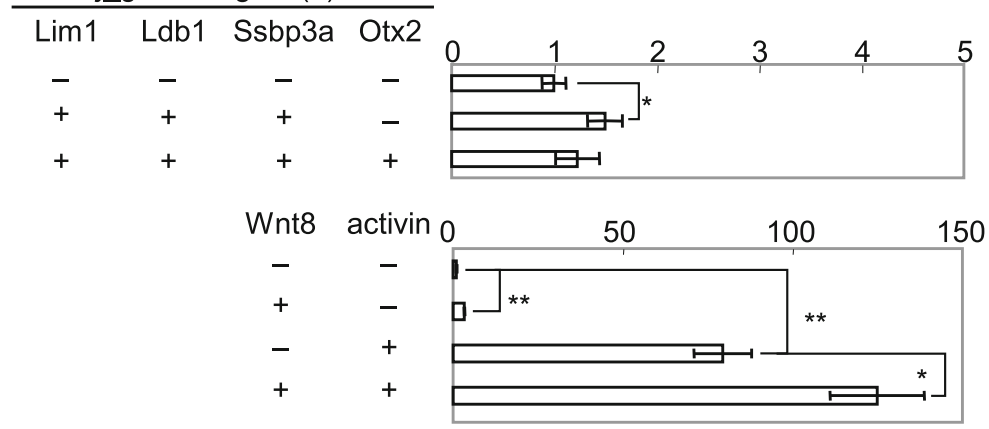

C

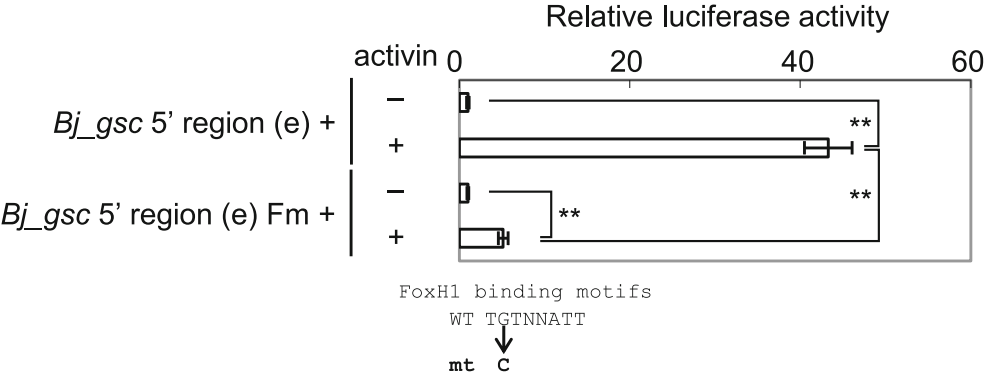

Fig. 5 Luciferase reporter analysis of the gsc $5^{\prime}$ region in Xenopus embryos. a Luciferase reporter assays of Bj_gsc $5^{\prime}$ regions for responsiveness to endogenous factors. Reporter constructs were injected into the animal pole (AP), ventral equatorial region (VER), or dorsal equatorial region (DER) at the four-cell stage to examine responsiveness of constructs to endogenous dorsal signals. Results were normalized with activity of embryos injected with reporter constructs into the animal pole. $\mathbf{b}$ Luciferase reporter assays of XI_gsc-U1 and the Bj_gsc 5' region for responsiveness to exogenous factors. Lim1/Ldb1/Ssbp3a strongly activated reporter gene expression through XI_gsc-U1 but only slightly through the Bj_gsc 5' region. While, Wnt and Nodal signaling synergistically activated reporter gene expression through the Bj_gsc $5^{\prime}$ region. c Luciferase reporter assays of Bj_gsc $5^{\prime}$ region with mutations of three FoxH1 motifs for responsiveness to Nodal signaling. The mutation construct greatly reduced responsiveness to activin, suggesting that Nodal/FoxH1 signaling directly regulates Bj_gsc through the $5^{\prime}$ region. See Fig. $4 \mathrm{~b}$ for details of the FoxH1 motif mutation. Reporter constructs were injected into the animal pole with combinations of mRNAs with dosages as follows: lim 1, 100 pg/embryo (XI_gsc-U1) or 50 pg/embryo (Bj_gsc 5' regions); Idb1, 100 pg/embryo (XI_gsc-U1) or 50 pg/embryo (Bj_gsc 5' regions); ssbp3, 100 pg/ embryo (XI_gsc-U1) or 50 pg/embryo (Bj_gsc 5' regions); otx2, 50 pg/embryo; wnt8, 25 pg/embryo; and activin A, 40 pg/embryo. Bars represent mean \pm s.e.m. ${ }^{*}, P<0.05 ;{ }^{*}, P<0.01$ ( $t$-test, two tailed) 

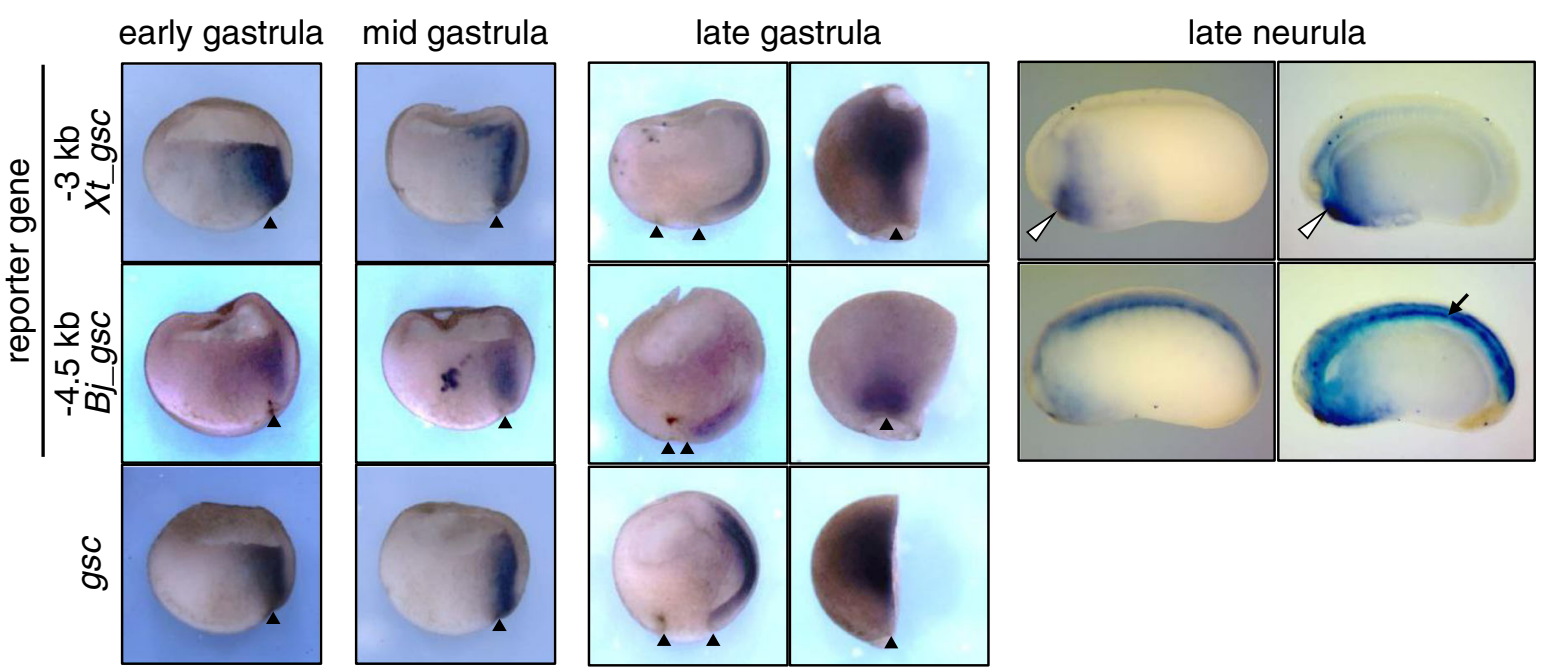

Fig. 6 Transgenic reporter analysis of the gsc $5^{\prime}$ region in Xenopus embryos. Transgenic reporter assays of the $-3 \mathrm{~kb}$ region of Xt_gsc and the $4.5 \mathrm{~kb}$ region of Bj_gsc in Xenopus embryos. Panels represent whole mount in situ hybridization of the reporter gene mVenus (first and second rows) or the endogenous gsc gene (third row) in dorso-ventral hemisections. Expression patterns were examined at the early (st. 10), middle (st. 11) and late (st. 12.5) gastrula stages and late-neurula stage (st. 23) as indicated. In right panels of late gastrula embryos, hemisections were represented in the dorsal view with anterior to the top. Other panels of gastrula are shown with animal to the top and dorsal to the right. Neurula embryos are shown with dorsal to the top and anterior to the left. Neurula embryos cleared in BB/BA solution are shown in right panels. Arrowheads, blastopore; open arrowheads, mouth; and arrows, notochord. Additional file 1: Figure S2 shows results in more detail (see Additional file 1)

expression in the organizer region. In late gastrula stage, the $-3 \mathrm{~kb}$ region of $X t \_g s c$ showed reporter expression in the head organizer, which recapitulates that of the endogenous $g s c$. By contrast, the $-4.5 \mathrm{~kb}$ region of $B j$ gsc showed reporter expression in the posterior mesoderm, but not in the head organizer. In neurula, the $-3 \mathrm{~kb}$ region of $X t$ gsc activates reporter genes around the mouth, whereas the $-4.5 \mathrm{~kb}$ region of Bj_gsc activates reporters in the notochord. In both constructs, reporter genes are frequently coactivated in the neural tissues and ventral ectoderm possibly due to the absence of CRMs for silencing in those regions (Additional file 1: Figure S2). Although the reporter expression was not restricted to the posterior notochord, expression patterns of the $-4.5 \mathrm{~kb} B j \_g s c$ reporter construct roughly resemble those of Bj_gsc in amphioxus gastrula to neurula (Fig. 1). The reporter expression in the anterior mesoderm derived from the organizer region disappeared in later stages possibly due to the absence of CRMs for maintaining the expression. Instead, the reporter gene was activated in the posterior mesoderm possibly through Nodal signaling because Nodal ligands are expressed in the posterior mesoderm in Xenopus [58-60]. Subsequently, it is maintained in the notochord of the neurula.

\section{Discussion}

In this study, we revealed the conservation of regulation for lim1 expression by Nodal/FoxH1 and chrd expression by Lim1 in the chordate gastrula organizer (Fig. 4).
We also found a distinct regulatory system of $g s c$ expression during gastrulation between amphioxus and vertebrates (Figs. 5 and 6). Among candidate CRMs in evolutionarily conserved sequences found by comparative genomics between B. lanceolatum, B. floridae, B. belcheri, and B. japonicum (Fig. 3), semi-conserved FoxH1 sites at the middle of Bj_lim1 intron 1 and conserved Lim1 sites in Bj_chrd intron 2 are supposed to be functional (Fig. 4). However, conserved Lim1 sites and bicoid sites in the $5^{\prime}$ upstream region of $B j \_g s c$ did not respond to Lim 1 and Otx2 (Fig. 5b). These results indicate that gene cascades in the head organizer of vertebrate are only partially conserved in amphioxus (Fig. 7a). Based on these findings and the comparison of gene expression patterns (Fig. 7b), we propose an evolutionary scenario for the head organizer as described below (Fig. 8).

In this scenario, Nodal is the ancestral organizerinducing signal, upregulating lim1, gsc, and chrd (Figs. 4 and 5, and [26]). By contrast, Wnt signal is thought to have been co-opted for organizer formation in ancient vertebrates to activate an additional organizer gene, like the homeobox gene siamois in Xenopus or bozozok in zebrafish $[12,15]$, because in amphioxus no dorsal accumulation of nuclear-catenin is observed in blastula to gastrula embryos and $\mathrm{LiCl}$ neither dorsalizes the embryo [61] nor activates gsc expression [62]. However, unexpectedly Bj_gsc was activated by Wnt8 to some extent in the reporter assay using Xenopus embryos (Fig. 5b). There are two possibilities to be tested: (i) in Xenopus 


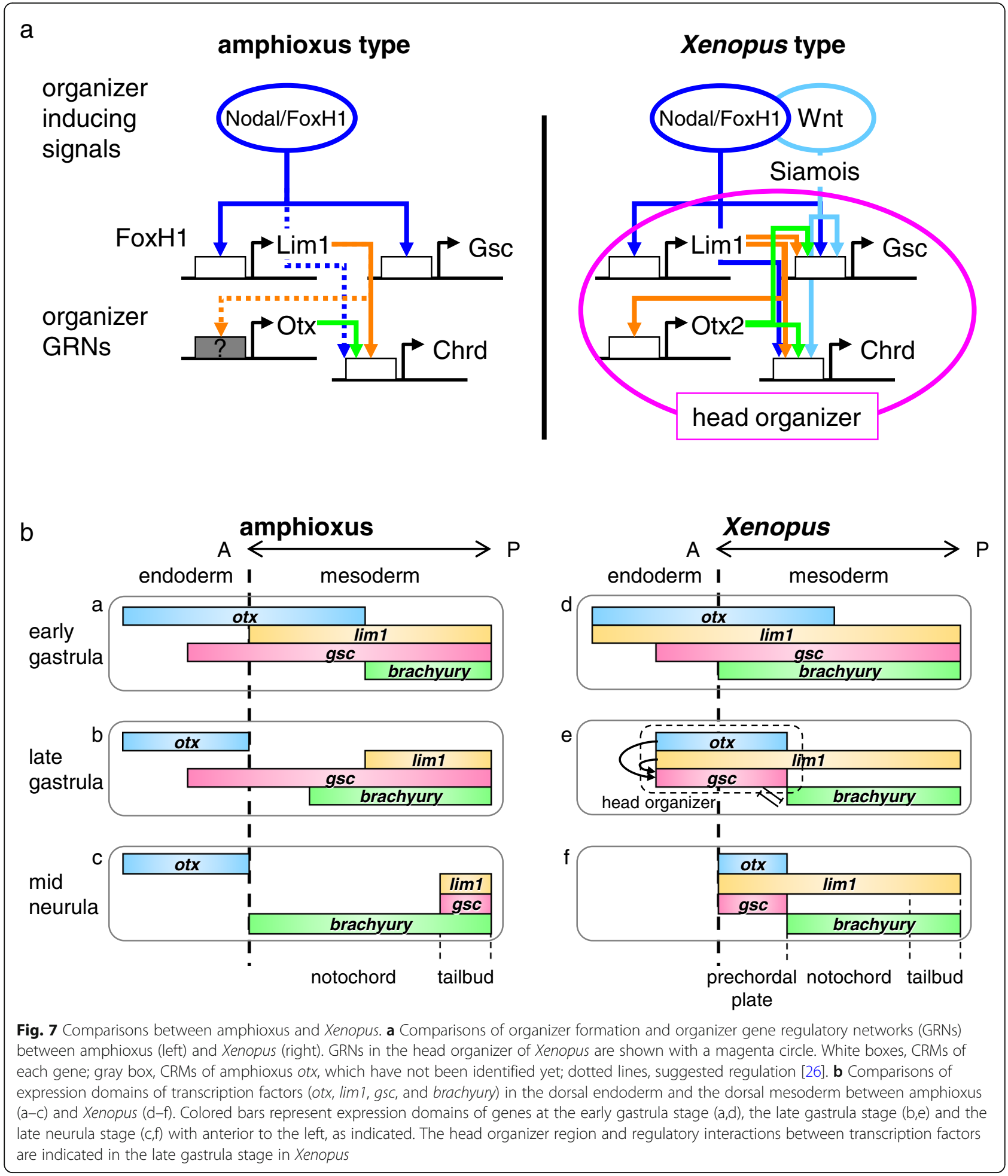

embryos, Wnt signaling induces chrd and Chrd relieves the Bj_gsc reporter from repression by Bmp signaling through Vent2 [45], or (ii) Wnt signaling enhances Nodal signaling through stabilizing Smad3 $[63,64]$. Together with our results, it is likely that the anterior expression domain of $g s c$ was acquired in the vertebrate lineage by co-option or creation of the CRM, which responds to Lim1 and Otx2. This CRM evolution may have been triggered by two rounds of whole-genome duplication before the emergence of vertebrates, which could have released them from evolutionary and developmental constraints of genes. 


\section{amphioxus-like chordate ancestor}

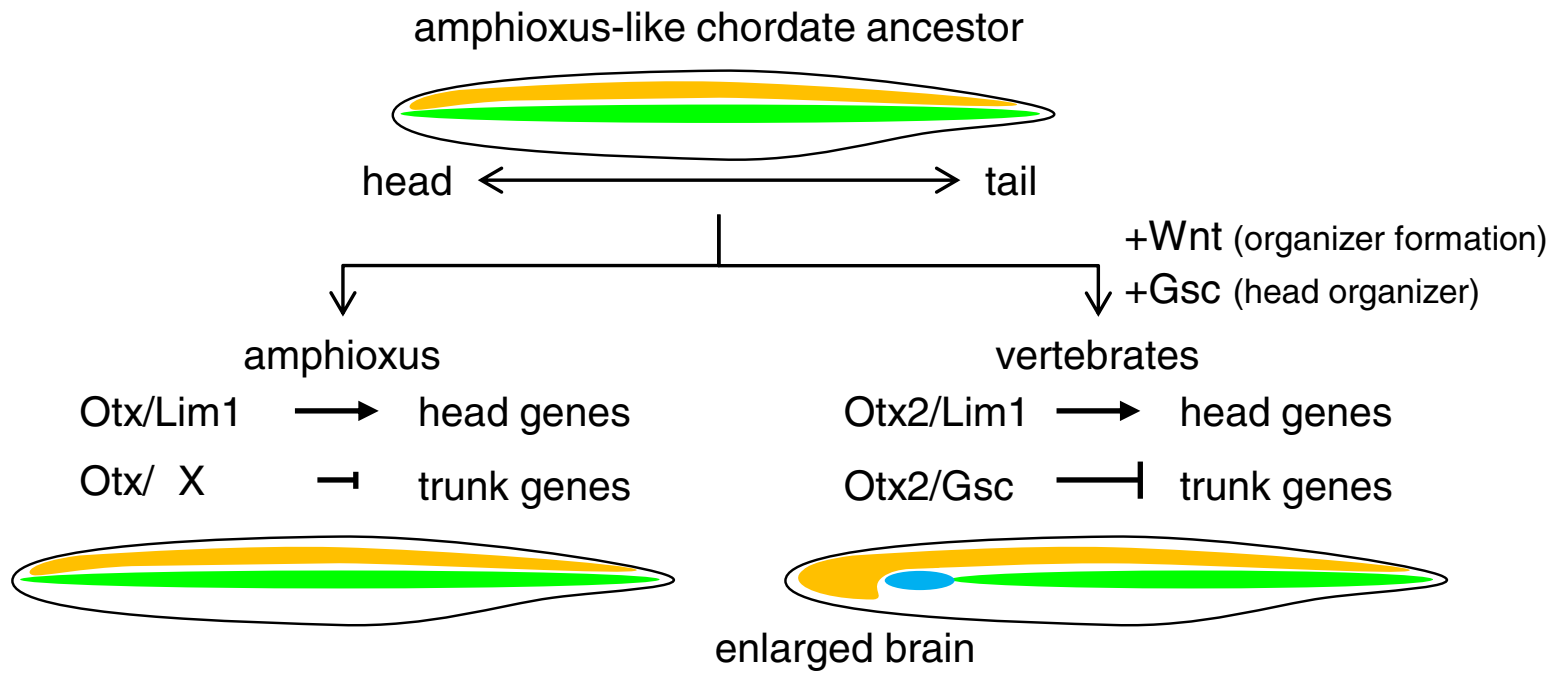

Fig. 8 Evolutionary scenario of the vertebrate head organizer. Assuming the amphioxus-like chordate ancestor, the vertebrate ancestor should have adopted Wnt signaling for organizer formation and coopted gsc as a target of Lim1 and Otx2 to form the anteriorly enlarged brain by converting the anterior presumptive notochordal cells to the prechordal plate. Schematics of body plans are shown with anterior to the left and dorsal to the top. Orange, brain and neural tube; green, notochord; blue, prechordal plate

What is the molecular consequence of $g s c$ cooption in the head organizer? A plausible answer comes from our recent study on the basis of genome-wide analysis using Xenopus embryos [21]. That study suggests that Otx2 upregulates head organizer genes, such as gsc, cerberus, and chrd, in cooperation with Lim1 through enhancers named "type I CRMs," whereas Otx2 downregulates trunk organizer genes, such as brachyury, wnt8a and not, with Gsc through silencers named "type II CRMs" [21]. The paper proposed that the bilaterian-conserved head selector, Otx, marks genes to be regulated in the head, and that its partner transcription factors that activate or repress Otx-marked genes can differ between organisms to make diverse head structures [21]. That is, the repression system of trunk organizer (notochord) genes in the anterior region of dorsal mesoderm may have been established by coopting gsc as a target gene for Lim1/Otx and by integrating Gsc as a repressive partner of the head selector, Otx. This possibility is also supported by previous reports. First, otx is expressed in the head region of all bilaterians and has a fundamental role to repress trunk genes such as hox genes $[65,66]$. Second, Gsc can bind to the same monomer binding motif as Otx, the bicoid site (TAATCY) [21, 39, 50, 67, 68], and has been predicted to form a heterodimer complex with Otx on the P3C site (TAATCNNATTA) [21, 69]. Third, even though gsc and brachyury in amphioxus are coexpressed in the dorsal mesoderm [25, 36, 70, 71], Gsc does not appear to repress brachyury, in contrast to vertebrate brachyury, which is repressed by Gsc and Otx2 [17, 18] (Fig. 7b). Our results also suggest that CRMs of brachyury in the vertebrate ancestor evolved to respond to a complex of Gsc and Otx for downregulation.

Incorporating our experimental data, we revisit the comparison of gene expression patterns between amphioxus and Xenopus (Fig. 7b), which is composed of previously reported expression data $[24,25,27,28,30-32,34$, $36,37,47,70-72]$. In amphioxus, otx, $\lim 1$, and, $g s c$ are all coexpressed in anterior mesoderm in the early gastrula stage (panel a in Fig. 7b), but possibly due to the absence of their regulatory interactions, otx, $\lim 1$, and $g s c$ are turned off in the anterior part until the mid-neurula stage (panel b,c). brachyury expression is overlapped with $\lim 1$ and $g S c$ in the posterior dorsal mesoderm in the early gastrula stage, and extends to the anterior during gastrulation. Then, the brachyury-expressing region develops into the notochord, and the posterior tip develops into the tail bud where $\lim 1, g s c$, and brachyury are expressed (panel c). In Xenopus, regulatory interactions between otx2, $\lim 1$, gsc, and brachyury have been established, thereby maintaining otx2, lim1, and gsc expression and repressing brachyury in the anterior part of mesoderm to form the head organizer (panels $\mathrm{d}$ and e). As a consequence, the anterior part develops into the prechordal plate, whereas the posterior part develops into the notochord (panel f). Thus, in the vertebrate ancestor, three major events are thought to have occurred. (i) $\lim 1$ and $o t x$ acquired type I CRMs (by mutations or cooption) to maintain their own expression through an auto-regulatory loop; (ii) gsc acquired type I CRMs to be upregulated by Lim1 and Otx2; and (iii) brachyury, as well as other posterior genes, acquired type II CRMs to be downregulated by Gsc and Otx2. The key event was to have created a new GRN for repression of 
trunk organizer genes by Gsc and Otx, which are maintained by Lim 1 and Otx in the anterior mesoderm. This GRN converts the notochordal mesoderm (trunk organizer) to the head organizer and later prechordal plate, leading to acquisition of the vertebrate-type head (Fig. 8). This hypothetical model should be addressed in the future by functional analyses of those transcription factors and reporter assays for potential CRMs in amphioxus embryos as performed previously [25, 26, 46, 73, 74].

Another aspect in the evolution of the vertebrate head mesoderm has also been examined experimentally by comparing vertebrates and amphioxus [47]. Onai et al. showed that mesodermal involution is a key developmental event to segregate dorsal mesoderm anteroposteriorly. In amphioxus, gastrulation occurs by simple invagination with little mesodermal involution, in contrast to vertebrate gastrulation which takes place through massive cell movements such as involution and convergent extension. Interestingly, inhibition of mesodermal involution in Xenopus embryos recapitulated amphioxus-like dorsal mesoderm formation, in which segregation of head-trunk organizers did not occur. Taken together with this study, both GRNs and cell movements during gastrulation must have been drastically rearranged in the vertebrate lineage to evolve the head.

How have the gastrula organizer and head organizer evolved among eumetazoans? Evolutionary data for the gastrula organizer has accumulated through studies of chordate outgroups, such as the sea anemone, Nematostella (reviewed in [75]). It has been reported that the blastopore lip of Nematostella has secondary axisinducing activity when transplanted into Nematostella embryos [76], and that many organizer-related genes including lim1, otx, foxa, and brachyury, but not gsc, are expressed around the blastopore [77-81]. Although a report with Nematostella showed that gsc is expressed in early gastrula endoderm [82], the detected expression was very weak and leaky compared to other ancestral organizer genes. These reports suggest that gsc was not an ancient organizer gene and that it was recruited to the organizer at least after bilaterians arose. In both polychaetes (Protostomes) and sea urchins (Deuterostomes), gsc and brachyury are coexpressed in the stomodaeum region (oral region) [83, 84], which is similar to the situation in the dorsal mesoderm of amphioxus. It was reported that knockdown of $g s c$ in sea urchin embryos eliminates brachyury expression in the oral ectoderm [85], which contrasts with the situation in vertebrates, suggesting that the deuterostome ancestor did not have the negative regulatory axis from Gsc to brachyury. On the other hand, no evidence was found for positive regulation of $g s c$ by Lim1 and Otx in any eumetazoans, except for vertebrates. Thus, it is probable that the positive regulatory axis from Lim1 and Otx to gsc and the negative regulatory axis from Gsc to brachyury is unique to vertebrates.

Neidert et al. [36] raised the question whether the ancestral chordate had (i) the amphioxus-type of head with the notochord and no anterior gsc expression or (ii) the vertebrate-type of head with the prechordal plate and anterior gsc expression. On the basis of our data and previous reports, we prefer the model (i) that the head organizer as well as the prechordal plate evolved in the vertebrate ancestor, which was initiated by cooption of gsc as a Lim1/Otx target gene (Fig. 8).

\section{Conclusions}

Gene regulatory networks conserved in chordates, and those specific to vertebrates, illustrate that rearrangement of CRMs for gsc is a key event for the evolution of the vertebrate head organizer. Co-option of gsc into the anterior mesoderm lead to further rearrangements of GRNs for head formation, resulting in the evolution of the vertebrate head with anteriorly enlarged brain.

\section{Materials and methods \\ Animals}

Adult male and female Xenopus laevis were purchased from Sato Breeder and Xenopus Yoshoku Kyozai (Japan), and maintained in our frog facility. Mature adult amphioxus, Branchiostoma japonicum, were collected in the coastal waters of the Enshu Nada Sea, Japan, during the breeding season.

Whole-mount in situ hybridization of amphioxus embryos The coding sequence of Bj_gsc was cloned into pGEM-T vector (Promega) by conjugating three exon sequences PCR-amplified from genomic DNA of $B$. japonicum using In-Fusion HD Cloning kit (Takara). Whole-mount in situ hybridization of amphioxus embryos was performed as described [86], using digoxigenin-labelled anti-sense probes for $B j g s c$, which was transcribed from linearized plasmids.

\section{Microinjection experiments in Xenopus embryos}

$X$. laevis fertilized eggs were dejellied and injected with mRNAs and reporter DNA. For mRNA synthesis, coding sequences of Xenopus genes used in this study were cloned into the pCSf107mT vector, which contains the SP6/T7 terminator [87]. Capped mRNA was synthesized using the mMESSAGE mMACHINE SP6 kit (Ambion). For luciferase assays, reporter plasmid DNA ( $50 \mathrm{pg} / \mathrm{em}$ bryo) with mRNAs for lim1, ldb1, ssbp3, otx2, siamois, wnt $8 a$, or activin $A$ (dosages are indicated in figure legends) were injected together into the animal pole region of both blastomeres at the two-cell stage. 


\section{Cloning of cis-regulatory regions}

The 5' region of Bj_gsc, Bj_lim1 intron 1 and Bj_chrd intron 2 were PCR-cloned with primers designed from the genome sequence of $B$. floridae (Joint Genome Institute, assembly version 1.0). Using sequences of obtained partial genomic DNA fragments, inverse PCR was performed to determine genomic sequences around the primers. Genomic sequences of B. japonicum have been deposited in the DDBJ as follows: 5' upstream region of $B j$ gsc (AB972409), the first intron of Bj_lim1 (AB972410), and the second intron of $B j_{-}$ chrd (AB972411). Second introns of Xt_chrd, $\mathrm{Xl}_{-}$ chrd.L and $\mathrm{Xl}$ _chrd.S were PCR-cloned with primers designed from the second and third exons using genomic DNA of N8A2 strain of X. tropicalis (the same strain as that used for the genome sequence in [88]) and J-strain of $X$. laevis (the same strain as that used for the genome sequence in [51]).

\section{Epigenetic data and vista plot}

ATAC-seq data and ChIP-seq data (H3K4me3, H3K27ac, and H3K27me3) of B. lanceolatum embryos [55] were visualized with UCSC genome browser track hubs (https:// genome-asia.ucsc.edu/cgi-bin/hgTracks?db=hub_78274_

BraLan2). Vista plots were generated with Vista tool (http://genome.lbl.gov/vista/index.shtml) using the B. lasceolatum genomic sequence as the $\mathrm{X}$-axis to combine with the epigenetic data. The following sequences were used for consensus binding motifs of transcription factors; $(\mathrm{C} /$ T)TAAT(T/G)(A/G) for Lim1, TAATC(C/T) for Otx2 (bicoid sites), TGTNNATT for FoxH1, and GTCTG for Smad [21, 39, 49, 50, 67, 68, 89-91].

\section{Luciferase assay using the Xenopus embryo}

Luciferase assays were performed as described [92]. To make reporter constructs for Bj_gsc (e, f, g in Figs. 3 and 5), 5' regions upstream from the start codon of Bj_gsc, which include the promoter, were connected to the start codon of the luciferase reporter gene in the pGL3 vector (Promega). Other constructs were made by inserting a genomic fragment into the pGL4.23 vector (Promega), which has an artificial, minimal promoter. Luciferase activity was analyzed at the gastrula stage (st 10.5-11). The mean and standard error were calculated by assaying five pools of three embryos for each injection sample. The results were normalized with activity of embryos injected with reporter constructs alone, and displayed as relative activity. Statistical significance was examined with Student's or Welch's $t$-test after $F$-test.

\section{Transgenic analysis in the Xenopus embryo}

Transgenesis was performed with sperm nuclear transplantation into unfertilized eggs with restriction- enzyme-mediated integration (REMI) methods as described [93-95]. Reporter constructs were made using a pGL4.23 derived vector, pGL4.23 mV, in which the luciferase gene was replaced with the coding sequence for the monomeric Venus fluorescent protein (mVenus, made by Y. Mii, Y. Honda and M. T.). A genomic fragment was inserted into the pGL4.23 mV vector as described for luciferase reporter constructs. Embryos were fixed at appropriate stages with MEMFA for $2 \mathrm{~h}$, dehydrated with ethanol and stored at $-20{ }^{\circ} \mathrm{C}$ overnight. Fixed embryos were mounted in a drop of $2 \%$ agarose and $0.3 \mathrm{M}$ sucrose in $1 \times$ PBS on a plastic dish [21,35]. Agarose-mounted embryos were bisected along the dorso-ventral axis, in which one half was used for detection of the reporter gene and the other half was used for detection of endogenous mRNAs. Hemisections were refixed with MEMFA for $1 \mathrm{~h}$ and dehydrated with methanol before whole mount in situ hybridization (WISH). WISH was performed as described [96] using digoxigenin-labelled anti-sense probes for mVenus, $\mathrm{Xl} \_g s c$, and $\mathrm{Xl}$ _chrd, which was transcribed from linearized plasmids. After treatment with alkaline phosphatase-conjugated antiDIG antibody (Roche; 1/2000 diluted), chromogenic reaction was performed with BM Purple (Roche). Stained embryos were refixed with Bouin solution (saturated picric acid solution: 37\% formaldehyde solution: acetic acid $=15: 5: 1$ ), bleached with peroxide, and cleared in BB/BA solution (benzyl benzoate: benzyl alcohol $=2: 1$ ) for observation.

\section{Additional file}

Additional file 1 Figure S1. Sequence alignment of CNSs examined in reporter assays a Alignment of a CNS in the middle of $\lim 1$ intron 1 of Branchiostoma species (analyzed in Fig. 4b). A Smad motif is completely conserved among Branchiostoma, but two FoxH1 motifs in Bj_lim1 are not conserved in other genomes. $\mathbf{b}$ Alignment of a CNS in the $5^{\prime}$ half of chrd intron 2 of Branchiostoma species (analyzed in Fig. 4d). In addition to two conserved Lim1 motifs, two FoxH1 motifs are also conserved among Branchiostoma. c Alignment of a CNS in the 3' half of chrd intron 2 of Branchiostoma species (analyzed in Fig. 4d). In addition to a conserved Lim1 motif, a FoxH1 motif is also conserved among Branchiostoma. $\mathbf{d}$ Alignment of a CNS in the $-1 \mathrm{~kb}$ region of gsc of Branchiostoma species. Three FoxH1 motifs are conserved among Branchiostoma. Figure S2. Details of transgenic reporter analysis of the gsc $5^{\prime}$ region in Xenopus embryos. Panels represent representative embryos in transgenic reporter assays. In early neurula, reporter gene of the $-0.5 \mathrm{~kb} X t_{-}$gsc construct is likely to be expressed in the prechordal plate, whereas $-4.5 \mathrm{~kb}$ Bj_gsc constcuts showed reporter expression in mesoderm (notochord) and ectoderm (neural tube) of the dorsal midline. In late neurula, $-3 \mathrm{~kb}$ Xt_gsc constructs often showed reporter expression in the neural tissue, but never in the notochord. Panels of cleared embryos are indicated with BB/BA at the right bottom. Embryos are shown in the same orientation as in Fig. 6, except for the right panel of early neurula expressing the $-4.5 \mathrm{~kb}$ Bj_gsc reporter, which is shown in the dorsal view with anterior to the left. Arrowheads, blastopore; open arrowheads, mouth; and arrows, notochord. The bottom table represents a summary of transgenic reporter assays ("expr." means expression). (PDF $188 \mathrm{~kb})$ 


\section{Acknowledgements}

We thank Steven D. Aird for technical editing of the manuscript. Branchiostoma japonicum embryos fixed for whole mount in situ hybridization were kindly provided from Dr. Jr-Kai Yu (Academia Sinica, Taiwan). Experiments using amphioxus embryos were helped by Drs. Yi-Jyun Luo and Tsai-Ming Lu (OIST, Japan). X. tropicalis genomic DNA was obtained from the National Bioresource Project of the MEXT, Japan.

\section{Authors' contributions}

YY performed experiments, analyzed the data, and wrote the paper. YT and KK prepared the genomic DNA and helped draft the manuscript. MT helped design experiments and draft the manuscript. All authors read and approved the final manuscript.

\section{Funding}

The work was supported in part by JSPS grants to YY (09 J02408, 12 J09531, 26650087 and 16 K21559), KK (20370026), and MT (22370075) and by Japanese Association of Marine Biology (JAMBIO, No. 23-76, YY).

\section{Availability of data and materials}

The datasets supporting the conclusions of this article are included within the article.

\section{Ethics approval and consent to participate}

All experiments with $X$. laevis were approved by the Animal Care and Use Committees in the University of Tokyo, Okinawa Institute of Science and Technology Graduate University (Nos. 2013-081 and 2016-169), and RIKEN (Project No. 2018-30).

\section{Consent for publication}

Not applicable.

\section{Competing interests}

The authors declare that they have no competing interests.

\section{Author details}

'Department of Biological Sciences, Graduate School of Science, University of Tokyo, 7-3-1 Hongo, Bunkyo-ku, Tokyo 113-0033, Japan. ${ }^{2}$ Marine Genomics Unit, Okinawa Institute of Science and Technology Graduate University, 1919-1 Tancha, Onna-son, Okinawa 904-0495, Japan. 'aboratory for Comprehensive Genomic Analysis, RIKEN Center for Integrative Medical Sciences, 1-7-22 Suehiro-cho, Tsurumi-ku, Yokohama 230-0045, Japan ${ }^{4}$ Center for Advance Marine Research, Ocean Research Institute, The University of Tokyo, 1-15-1, Minamidai, Nakano-ku, Tokyo 164-8639, Japan. ${ }^{5}$ Present address: Cell Resource Center for Biomedical Research, Institute of Development, Aging and Cancer (IDAC), Tohoku University, 4-1 Seiryo-machi, Aoba-ku, Sendai, Miyagi 980-8575, Japan. ${ }^{6}$ Present address: SIRC, Teikyo University, 2-11-1, Itabashi-ku, Tokyo 173-8605, Japan. ${ }^{7}$ Present address: Department of Biological Sciences, Faculty of Science and Engineering, Chuo University, 1-13-27 Kasuga, Bunkyo-ku, Tokyo 112-8551, Japan.

Received: 30 December 2018 Accepted: 12 July 2019

\section{Published online: 02 August 2019}

\section{References}

1. Northcutt RG, Gans C. The genesis of neural crest and epidermal placodes: a reinterpretation of vertebrate origins. Q Rev Biol. 1983;58(1):1-28.

2. Glenn NR. The new head hypothesis revisited. J Exp Zool B Mol Dev Evol. 2005:304(4):274-97.

3. Holland LZ, Short S. Gene duplication, co-option and recruitment during the origin of the vertebrate brain from the invertebrate chordate brain. Brain Behav Evol. 2008;72(2):91-105.

4. Bertrand S, Escriva H. Evolutionary crossroads in developmental biology: amphioxus. Development. 2011;138(22):4819-30.

5. Holland LZ. Chordate roots of the vertebrate nervous system: expanding the molecular toolkit. Nat Rev Neurosci. 2009:10(10):736-46

6. Pani AM, Mullarkey EE, Aronowicz J, Assimacopoulos S, Grove EA, Lowe CJ. Ancient deuterostome origins of vertebrate brain signalling centres. Nature. 2012;483(7389):289-94.

7. Lowe CJ, Clarke DN, Medeiros DM, Rokhsar DS, Gerhart J. The deuterostome context of chordate origins. Nature. 2015;520(7548):456-65.
8. Horie R, Hazbun A, Chen K, Cao C, Levine M, Horie T. Shared evolutionary origin of vertebrate neural crest and cranial placodes. Nature. 2018. 560(7717):228-32.

9. Robb L, Tam PP. Gastrula organiser and embryonic patterning in the mouse. Semin Cell Dev Biol. 2004;15(5):543-54.

10. De Robertis EM, Larrain J, Oelgeschlager M, Wessely O. The establishment of Spemann's organizer and patterning of the vertebrate embryo. Nat Rev Genet. 2000;1(3):171-81.

11. Gore AV, Maegawa S, Cheong A, Gilligan PC, Weinberg ES, Sampath K. The zebrafish dorsal axis is apparent at the four-cell stage. Nature. 2005; 438(7070):1030-5.

12. Schier AF, Talbot WS. Molecular genetics of axis formation in zebrafish. Annu Rev Genet. 2005:39:561-613.

13. Hagos EG, Fan X, Dougan ST. The role of maternal Activin-like signals in zebrafish embryos. Dev Biol. 2007;309(2):245-58.

14. Niehrs C. Regionally specific induction by the Spemann-Mangold organizer. Nat Rev Genet. 2004;5(6):425-34.

15. Heasman J. Patterning the early Xenopus embryo. Development. 2006; 133(7):1205-17.

16. de Souza FS, Niehrs C. Anterior endoderm and head induction in early vertebrate embryos. Cell Tissue Res. 2000;300(2):207-17.

17. Artinger M, Blitz I, Inoue K, Tran U, Cho KW. Interaction of goosecoid and brachyury in Xenopus mesoderm patterning. Mech Dev. 1997:65(1-2):187-96.

18. Latinkic BV, Umbhauer M, Neal KA, Lerchner W, Smith JC, Cunliffe V. The Xenopus Brachyury promoter is activated by FGF and low concentrations of activin and suppressed by high concentrations of activin and by pairedtype homeodomain proteins. Genes Dev. 1997;11(23):3265-76.

19. Yao J, Kessler DS. Goosecoid promotes head organizer activity by direct repression of Xwnt8 in Spemann's organizer. Development. 2001;128(15): 2975-87.

20. Sander V, Reversade B, De Robertis EM. The opposing homeobox genes Goosecoid and Vent1/2 self-regulate Xenopus patterning. EMBO J. 2007; 26(12):2955-65.

21. Yasuoka Y, Suzuki Y, Takahashi S, Someya H, Sudou N, Haramoto Y, et al. Occupancy of tissue-specific cis-regulatory modules by OtX2 and TLE/ Groucho for embryonic head specification. Nat Commun. 2014:5:4322.

22. Herrmann BG, Kispert A. The T genes in embryogenesis. Trends Genet. 1994; 10(8):280-6.

23. Garcia-Fernandez J, D'Aniello S, Escriva H. Organizing chordates with an organizer. BioEssays. 2007;29(7):619-24.

24. Yu JK, Satou Y, Holland ND, Shin IT, Kohara Y, Satoh N, et al. Axial patterning in cephalochordates and the evolution of the organizer. Nature. 2007; 445(7128):613-7.

25. Onai T, Lin HC, Schubert M, Koop D, Osborne PW, Alvarez S, et al. Retinoic acid and Wnt/beta-catenin have complementary roles in anterior/posterior patterning embryos of the basal chordate amphioxus. Dev Biol. 2009;332(2): 223-33.

26. Onai T, Yu JK, Blitz IL, Cho KW, Holland LZ. Opposing nodal/Ng1 and BMP signals mediate axial patterning in embryos of the basal chordate amphioxus. Dev Biol. 2010;344(1):377-89.

27. Langeland JA, Holland LZ, Chastain RA, Holland ND. An amphioxus LIMhomeobox gene, AmphiLim1/5, expressed early in the invaginating organizer region and later in differentiating cells of the kidney and central nervous system. Int J Biol Sci. 2006;2(3):110-6.

28. Taira M, Jamrich M, Good PJ, Dawid IB. The LIM domain-containing homeo box gene Xlim-1 is expressed specifically in the organizer region of Xenopus gastrula embryos. Genes Dev. 1992;6(3):356-66.

29. Sasai Y, Lu B, Steinbeisser H, Geissert D, Gont LK, De Robertis EM. Xenopus chordin: a novel dorsalizing factor activated by organizer-specific homeobox genes. Cell. 1994;79(5):779-90.

30. Taira M, Otani H, Jamrich M, Dawid IB. Expression of the LIM class homeobox gene Xlim-1 in pronephros and CNS cell lineages of Xenopus embryos is affected by retinoic acid and exogastrulation. Development. 1994;120(6):1525-36.

31. Blitz IL, Cho KW. Anterior neurectoderm is progressively induced during gastrulation: the role of the Xenopus homeobox gene orthodenticle. Development. 1995;121(4):993-1004.

32. Pannese M, Polo C, Andreazzoli M, Vignali R, Kablar B, Barsacchi G, et al. The Xenopus homologue of Otx2 is a maternal homeobox gene that demarcates and specifies anterior body regions. Development. 1995;121(3): 707-20. 
33. Toyama R, Curtiss PE, Otani H, Kimura M, Dawid IB, Taira M. The LIM class homeobox gene lim5: implied role in CNS patterning in Xenopus and zebrafish. Dev Biol. 1995;170(2):583-93.

34. Kuroda H, Hayata T, Eisaki A, Asashima M. Cloning a novel developmental regulating gene, Xot $\times 5$ : its potential role in anterior formation in Xenopus laevis. Develop Growth Differ. 2000:42(2):87-93.

35. Sudou N, Yamamoto S, Ogino H, Taira M. Dynamic in vivo binding of transcription factors to cis-regulatory modules of cer and gsc in the stepwise formation of the Spemann-Mangold organizer. Development. 2012;139:1651-61.

36. Neidert AH, Panopoulou G, Langeland JA. Amphioxus goosecoid and the evolution of the head organizer and prechordal plate. Evol Dev. 2000;2(6): 303-10.

37. Cho KW, Blumberg B, Steinbeisser H, De Robertis EM. Molecular nature of Spemann's organizer: the role of the Xenopus homeobox gene goosecoid. Cell. 1991;67(6):1111-20.

38. Watabe T, Kim S, Candia A, Rothbacher U, Hashimoto C, Inoue K, et al. Molecular mechanisms of Spemann's organizer formation: conserved growth factor synergy between Xenopus and mouse. Genes Dev. 1995; 9(24):3038-50.

39. Mochizuki T, Karavanov AA, Curtiss PE, Ault KT, Sugimoto N, Watabe T, et al. Xlim-1 and LIM domain binding protein 1 cooperate with various transcription factors in the regulation of the goosecoid promoter. Dev Biol. 2000;224(2):470-85.

40. Koide T, Hayata T, Cho KW. Xenopus as a model system to study transcriptional regulatory networks. Proc Natl Acad Sci U S A. 2005;102(14): 4943-8.

41. Charney RM, Paraiso KD, Blitz IL, Cho KWY. A gene regulatory program controlling early Xenopus mesendoderm formation: network conservation and motifs. Semin Cell Dev Biol. 2017:66:12-24.

42. Laurent MN, Blitz IL, Hashimoto C, Rothbacher U, Cho KW. The Xenopus homeobox gene twin mediates Wnt induction of goosecoid in establishment of Spemann's organizer. Development. 1997;124(23):4905-16.

43. Ring C, Ogata S, Meek L, Song J, Ohta T, Miyazono K, et al. The role of a Williams-Beuren syndrome-associated helix-loop-helix domain-containing transcription factor in activin/nodal signaling. Genes Dev. 2002;16(7):820-35.

44. Trindade $M$, Tada M, Smith JC. DNA-binding specificity and embryological function of Xom (Xvent-2). Dev Biol. 1999;216(2):442-56.

45. Kozmikova I, Smolikova J, Vlcek C, Kozmik Z. Conservation and diversification of an ancestral chordate gene regulatory network for dorsoventral patterning. PLoS One. 2011;6(2):e14650.

46. Kozmikova I, Candiani S, Fabian P, Gurska D, Kozmik Z. Essential role of bmp signaling and its positive feedback loop in the early cell fate evolution of chordates. Dev Biol. 2013;382(2):538-54.

47. Onai $T$, Aramaki $T$, Inomata H, Hirai $T$, Kuratani S. Ancestral mesodermal reorganization and evolution of the vertebrate head. Zoological Lett. 2015;1:29

48. Rebbert ML, Dawid IB. Transcriptional regulation of the Xlim-1 gene by activin is mediated by an element in intron I. Proc Natl Acad Sci U S A. 1997;94(18):9717-22

49. Watanabe M, Rebbert ML, Andreazzoli M, Takahashi N, Toyama R, Zimmerman S, et al. Regulation of the Lim-1 gene is mediated through conserved FAST-1/FoxH1 sites in the first intron. Dev Dyn. 2002;225(4): 448-56.

50. Yamamoto $\mathrm{S}$, Hikasa $\mathrm{H}$, Ono H, Taira M. Molecular link in the sequential induction of the Spemann organizer: direct activation of the cerberus gene by Xlim-1, Xotx2, mix.1, and Siamois, immediately downstream from nodal and Wnt signaling. Dev Biol. 2003;257(1):190-204.

51. Session AM, Uno Y, Kwon T, Chapman JA, Toyoda A, Takahashi S, et al. Genome evolution in the allotetraploid frog Xenopus laevis. Nature. 2016; 538(7625):336-43.

52. Charney RM, Forouzmand E, Cho JS, Cheung J, Paraiso KD, Yasuoka Y, et al. Foxh1 occupies cis-regulatory modules prior to dynamic transcription factor interactions controlling the Mesendoderm gene program. Dev Cell. 2017; 40(6):595-607 e4.

53. Putnam NH, Butts $T$, Ferrier DE, Furlong RF, Hellsten U, Kawashima $T$, et al. The amphioxus genome and the evolution of the chordate karyotype. Nature. 2008:453(7198):1064-71

54. Huang S, Chen Z, Yan X, Yu T, Huang G, Yan Q, et al. Decelerated genome evolution in modern vertebrates revealed by analysis of multiple lancelet genomes. Nat Commun. 2014;5:5896.
55. Marletaz F, Firbas PN, Maeso I, Tena JJ, Bogdanovic O, Perry M, et al. Amphioxus functional genomics and the origins of vertebrate gene regulation. Nature. 2018;564(7734):64-70.

56. Berman BP, Pfeiffer BD, Laverty TR, Salzberg SL, Rubin GM, Eisen MB, et al. Computational identification of developmental enhancers: conservation and function of transcription factor binding-site clusters in Drosophila melanogaster and Drosophila pseudoobscura. Genome Biol. 2004;5(9):R61.

57. Visel A, Blow MJ, Li Z, Zhang T, Akiyama JA, Holt A, et al. ChIP-seq accurately predicts tissue-specific activity of enhancers. Nature. 2009; 457(7231):854-8.

58. Joseph EM, Melton DA. Xnr4: a Xenopus nodal-related gene expressed in the Spemann organizer. Dev Biol. 1997;184(2):367-72.

59. Onuma Y, Yeo CY, Whitman M. XCR2, one of three Xenopus EGF-CFC genes, has a distinct role in the regulation of left-right patterning. Development. 2006:133(2):237-50.

60. Zamparini AL, Watts T, Gardner CE, Tomlinson SR, Johnston Gl, Brickman JM Hex acts with beta-catenin to regulate anteroposterior patterning via a Groucho-related co-repressor and nodal. Development. 2006;133(18):3709-22.

61. Holland LZ, Panfilio KA, Chastain R, Schubert M, Holland ND. Nuclear betacatenin promotes non-neural ectoderm and posterior cell fates in amphioxus embryos. Dev Dyn. 2005:233(4):1430-43.

62. Yasui K, Li G, Wang Y, Saiga H, Zhang P, Aizawa S. Beta-catenin in early development of the lancelet embryo indicates specific determination of embryonic polarity. Develop Growth Differ. 2002;44(6):467-75.

63. Guo X, Ramirez A, Waddell DS, Li Z, Liu X, Wang XF. Axin and GSK3- control Smad3 protein stability and modulate TGF- signaling. Genes Dev. 2008;22(1): 106-20.

64. Hua F, Zhou J, Liu J, Zhu C, Cui B, Lin H, et al. Glycogen synthase kinase3beta negatively regulates TGF-beta1 and angiotensin II-mediated cellular activity through interaction with Smad3. Eur J Pharmacol. 2010;644(1-3): 17-23.

65. Arendt D, Nubler-Jung K. Common ground plans in early brain development in mice and flies. BioEssays. 1996;18(3):255-9.

66. Reichert $\mathrm{H}$, Simeone A. Developmental genetic evidence for a monophyletic origin of the bilaterian brain. Philos Trans R Soc Lond Ser B Biol Sci. 2001;356(1414):1533-44.

67. Berger MF, Badis G, Gehrke AR, Talukder S, Philippakis AA, Pena-Castillo L, et al. Variation in homeodomain DNA binding revealed by high-resolution analysis of sequence preferences. Cell. 2008;133(7):1266-76.

68. Noyes MB, Christensen RG, Wakabayashi A, Stormo GD, Brodsky MH, Wolfe SA. Analysis of homeodomain specificities allows the family-wide prediction of preferred recognition sites. Cell. 2008;133(7):1277-89.

69. Wilson D, Sheng G, Lecuit T, Dostatni N, Desplan C. Cooperative dimerization of paired class homeo domains on DNA. Genes Dev. 1993; 7(11):2120-34

70. Terazawa K, Satoh N. Formation of the chordamesoderm in the amphioxus embryo: analysis with Brachyury and fork head/HNF-3 genes. Dev Genes Evol. 1997;207(1):1-11.

71. Zhang SC, Holland ND, Holland LZ. Topographic changes in nascent and early mesoderm in amphioxus embryos studied by Dil labeling and by in situ hybridization for a Brachyury gene. Dev Genes Evol. 1997; 206(8):532-5.

72. Holland PW, Koschorz B, Holland LZ, Herrmann BG. Conservation of Brachyury (T) genes in amphioxus and vertebrates: developmental and evolutionary implications. Development. 1995:121(12):4283-91.

73. Holland LZ, Albalat R, Azumi K, Benito-Gutierrez E, Blow MJ, Bronner-Fraser $M$, et al. The amphioxus genome illuminates vertebrate origins and cephalochordate biology. Genome Res. 2008;18(7):1100-11.

74. Yue JX, Kozmikova I, Ono H, Nossa CW, Kozmik Z, Putnam NH, et al. Conserved noncoding elements in the Most distant genera of cephalochordates: the goldilocks principle. Genome Biol Evol. 2016:8(8): 2387-405.

75. Yasuoka $Y$, Taira M. The molecular basis of the gastrula organizer in amphibians and cnidarians. In: Kobayashi K, Kitano T, Iwao Y, Kondo M, editors. Reproductive and developmental strategies: the continuity of life. Tokyo: Springer Japan; 2018. p. 667-708.

76. Kraus Y, Fritzenwanker JH, Genikhovich G, Technau U. The blastoporal organiser of a sea anemone. Curr Biol. 2007;17(20):R874-6.

77. Scholz CB, Technau U. The ancestral role of Brachyury: expression of NemBra1 in the basal cnidarian Nematostella vectensis (Anthozoa). Dev Genes Evol. 2003;212(12):563-70. 
78. Fritzenwanker JH, Saina M, Technau U. Analysis of forkhead and snail expression reveals epithelial-mesenchymal transitions during embryonic and larval development of Nematostella vectensis. Dev Biol. 2004;275(2):389-402.

79. Matus DQ, Pang K, Marlow H, Dunn CW, Thomsen GH, Martindale MQ. Molecular evidence for deep evolutionary roots of bilaterality in animal development. Proc Natl Acad Sci U S A. 2006;103(30):11195-200.

80. Mazza ME, Pang K, Martindale MQ, Finnerty JR. Genomic organization, gene structure, and developmental expression of three clustered otx genes in the sea anemone Nematostella vectensis. J Exp Zoolog B Mol Dev Evol. 2007; 308(4):494-506.

81. Yasuoka Y, Kobayashi M, Kurokawa D, Akasaka K, Saiga H, Taira M. Evolutionary origins of blastoporal expression and organizer activity of the vertebrate gastrula organizer gene $\mathrm{lhx} 1$ and its ancient metazoan paralog Ihx3. Development. 2009;136(12):2005-14.

82. Rottinger $E$, Dahlin P, Martindale MQ. A framework for the establishment of a cnidarian gene regulatory network for "endomesoderm" specification: the inputs of ss-catenin/TCF signaling. PLoS Genet. 2012;8(12):e1003164.

83. Arendt $\mathrm{D}$, Technau U, Wittbrodt J. Evolution of the bilaterian larval foregut. Nature. 2001;409(6816):81-5.

84. Duboc V, Rottinger E, Besnardeau L, Lepage T. Nodal and BMP2/4 signaling organizes the oral-aboral axis of the sea urchin embryo. Dev Cell. 2004;6(3): 397-410.

85. Saudemont A, Haillot E, Mekpoh F, Bessodes N, Quirin M, Lapraz F, et al, Ancestral regulatory circuits governing ectoderm patterning downstream of nodal and BMP2/4 revealed by gene regulatory network analysis in an echinoderm. PLoS Genet. 2010;6(12):e1001259.

86. Yu JK, Holland LZ. Amphioxus whole-mount in situ hybridization. Cold Spring Harb Protoc. 2009;2009(9):pdb.prot5286.

87. Mii Y, Taira M. Secreted frizzled-related proteins enhance the diffusion of Wnt ligands and expand their signalling range. Development. 2009;136(24): 4083-8.

88. Hellsten U, Harland RM, Gilchrist MJ, Hendrix D, Jurka J, Kapitonov V, et al. The genome of the Western clawed frog Xenopus tropicalis. Science. 2010; 328(5978):633-6.

89. Massague J, Wotton D. Transcriptional control by the TGF-beta/Smad signaling system. EMBO J. 2000;19(8):1745-54.

90. Massague J, Seoane J, Wotton D. Smad transcription factors. Genes Dev. 2005;19(23):2783-810.

91. Fukuda M, Takahashi S, Haramoto Y, Onuma Y, Kim YJ, Yeo CY, et al. Zygotic VegT is required for Xenopus paraxial mesoderm formation and is regulated by nodal signaling and Eomesodermin. Int J Dev Biol. 2010;54(1):81-92.

92. Yasuoka Y. Taira M. Cold Spring Harb Protoc: Microinjection of DNA Constructs into Xenopus Embryos for Gene Misexpression and cisRegulatory Module Analysis; 2018.

93. Ishibashi S, Kroll KL, Amaya E. Generation of Transgenic Xenopus laevis: III. Sperm Nuclear Transplantation. CSH Protoc. 2007;2007:pdb.prot4840.

94. Ishibashi S, Kroll KL, Amaya E. Generation of Transgenic Xenopus laevis: II. Sperm Nuclei Preparation. CSH Protoc. 2007;2007:pdb.prot4839.

95. Ishibashi S, Kroll KL, Amaya E. Generation of Transgenic Xenopus laevis: I. High-Speed Preparation of Egg Extracts. CSH Protoc. 2007;2007:pdb. prot4838.

96. Harland RM. In situ hybridization: an improved whole-mount method for Xenopus embryos. Methods Cell Biol. 1991;36:685-95.

\section{Publisher's Note}

Springer Nature remains neutral with regard to jurisdictional claims in published maps and institutional affiliations.

Ready to submit your research? Choose BMC and benefit from:

- fast, convenient online submission

- thorough peer review by experienced researchers in your field

- rapid publication on acceptance

- support for research data, including large and complex data types

- gold Open Access which fosters wider collaboration and increased citations

- maximum visibility for your research: over $100 \mathrm{M}$ website views per year

At BMC, research is always in progress.

Learn more biomedcentral.com/submissions 